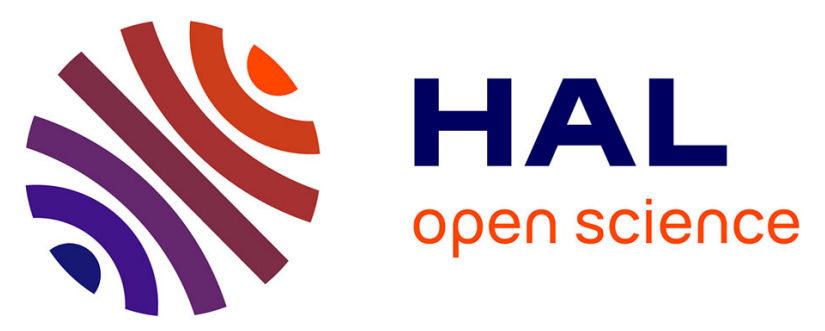

\title{
Les causes du suicide: pathologie individuelle ou sociale? Durkheim, Halbwachs et les psychiatres de leur temps (1830-1930)
}

Laurent Mucchielli, Marc Renneville

\section{- To cite this version:}

Laurent Mucchielli, Marc Renneville. Les causes du suicide: pathologie individuelle ou sociale? Durkheim, Halbwachs et les psychiatres de leur temps (1830-1930). Déviance et Société, 1998, 22 (1), pp.3 - 36. 10.3406/ds.1998.1647 . halshs-00130224

\section{HAL Id: halshs-00130224 \\ https://shs.hal.science/halshs-00130224}

Submitted on 1 Nov 2016

HAL is a multi-disciplinary open access archive for the deposit and dissemination of scientific research documents, whether they are published or not. The documents may come from teaching and research institutions in France or abroad, or from public or private research centers.
L'archive ouverte pluridisciplinaire HAL, est destinée au dépôt et à la diffusion de documents scientifiques de niveau recherche, publiés ou non, émanant des établissements d'enseignement et de recherche français ou étrangers, des laboratoires publics ou privés.

\section{(이) $\$$}

Distributed under a Creative Commons Attribution - NonCommercial - NoDerivatives 44.0 


\section{Les causes du suicide : pathologie individuelle ou sociale?} Durkheim, Halbwachs et les psychiatres de leur temps (1830-1930) Laurent Mucchielli, Marc Renneville

\section{Citer ce document / Cite this document :}

Mucchielli Laurent, Renneville Marc. Les causes du suicide : pathologie individuelle ou sociale? Durkheim, Halbwachs et les psychiatres de leur temps (1830-1930). In: Déviance et société. 1998 - Vol. 22 - N¹. pp. 3-36;

doi : $10.3406 /$ ds. 1998.1647

http://www.persee.fr/doc/ds_0378-7931_1998_num_22_1_1647

Document généré le 06/06/2016 


\begin{abstract}
The article questions the link between individuals and society and the ideas of psychological causality set out by Durkheim in Suicide, and puts them into historical perspective, considering previous readings and the critical revisionist perspective by Halb- wachs in 1930. First, we consider the ways in which Durkheim interpreted psychiatric theories which explained social facts through individual pathology. We then show that this Durkheimian construction involves the rejection of individual motive and replaces it by a different psychological interpretation which is based on socially determined unconscious mechanisms. We underline the fact that this theoretical construction had several difficulties even in relation to the empirical findings of the period and that it leads us to question the Durkheimian typology of suicide. Finally, we consider the way in which, some 30 years later, Halbwachs was able largely to resolve these difficulties by abandoning the theoretical axioms which Durkheim had placed at the heart of sociological analysis.
\end{abstract}

De auteurs van dit artikel bestuderen de verhouding individu / maatschappij alsook het psychische causaliteitsmodel zoals bedacht door Durkheim in zijn werk «Le Suicide». Ze plaatsen dit werk in een historisch perspectief ten aanzien van enerzijds de interpretaties (gedachtegoed) die het werk vooraf gingen en anderzijds vertrekkende van de kritische herlezing verricht door Halbwachs in 1930. In eerste instantie wordt door de auteurs in detail de manier besproken waarop Durkheim de psychiatrische stellingen afweert. Stellingen die volgens hem een sociaal feit verklaren door individuele pathologie (ziekten-kunde). Vervolgens wordt aangetoond dat de opstelling van Durkheim de verwerping van de analyse van individuele motieven impliceert en deze theoretische constructie met een aantal tegenkantingen te maken krijgt als ze wordt geconfronteerd met empirische gegevens uit de tijd. Uiteindelijk brengt dit met zich mee dat men zich vragen gaat stellen inzake de gegrondheid van Durkheim's zelfmoordtypologie. Tenslotte wordt in het artikel de manier bestudeerd waarop meer dan 30 jaar later Halbwachs erin slaagt deze moeilijk-heden op te lossen door afstand te nemen van de theoretische axioma's die Durkheim had geplaatst in het kader van de sociologische analyse.

\title{
Zusammenfassung
}

Dieser Artikel behandelt in historischer Perspektive die Beziehung zwischen Indivi- duum und Gesellschaft sowie das Modell psychischer Ursachen, wie es von Durkheim in seiner Selbstmordstudie entwickelt wurde. Dazu wird dieses Modell einerseits in Beziehung gesetzt zum Stand der Interpretationen, die inm vorausgingen und andererseits zu einer kritischen Lesart, wie sie von Halbwachs 1930 entwickelt worden ist. Zunächst gehen wir im Detail auf die Analyse von Durkheim ein, in der er psychiatrische Thesen behandelt, die fur inn eine soziale Tatsache iiber individuelle Pathologien erklären. Wir zeigen dann, daß die durkheimsche Konstruktion eine Zuruckweisung individueller Motive impliziert, welche durch eine andere psychologische Interpretation ersetzt werden, die sich auf sozial determinierte unbewußte Mechanismen grundet. Dabei unterstreichen wir, daß diese theoretische Konstruktion auf einige Schwierigkeiten bei der Konfrontation mit den empirischen Daten der damaligen Zeit stößt und den Wert der durkheimschen Selbst- mordtypologie grundsätzlich in Frage stellt. Schließlich untersuchen wir die Art, in der es Halbwachs dreißig Jahre später gelang, diese Probleme eher zu lösen, indem er auf die theoretischen Postulate, die Durkheim ins Zentrum der soziologischen Analyse stellte, verzichtete.

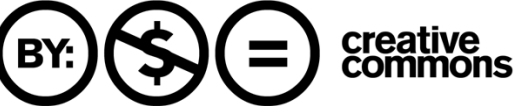




\title{
LES CAUSES DU SUICIDE: PATHOLOGIE INDIVIDUELLE OU SOCIALE? DURKHEIM, HALBWACHS ET LES PSYCHIATRES DE LEUR TEMPS (1830-1930)
}

\author{
L. MUCCHIELLI *, M. RENNEVILLE **
}

Cet article interroge le rapport individu / société ainsi que le modèle de causalité psychique conçus par Durkheim dans le Suicide, et les met en perspective historique au regard d'une part de l'état des interprétations l'ayant précédé, d'autre part de la relecture critique opérée par Halbwachs en 1930. Nous revenons d'abord en détail sur la façon dont Durkheim écarte les thèses psychiatriques qui, selon lui, expliquent un fait social par des pathologies individuelles. Nous montrons ensuite que la construction durkheimienne implique le rejet de l'analyse des motifs individuels pour les remplacer par une autre interprétation psychologique fondée sur l'existence de mécanismes inconscients socialement déterminés. Au passage, nous soulignons que cette construction théorique rencontre quelques difficultés dans la confrontation avec les données empiriques de l'époque, et qu'elle amène en définitive à s'interroger sur le bien-fondé de la typologie durkheimienne des suicides. Enfin, nous examinons la façon dont, plus de trente ans après, Halbwachs parvient largement à résoudre ces difficultés en abandonnant les postulats théoriques que Durkheim avait placés au caeur de l'analyse sociologique.

Mots-Clés: Suicide - Théorie sociologiQue - Histoire de la pSychiatrie HistoIRE DE LA SOCIOLOGIE - EPISTÉMOLOGIE

Bien qu'il ait été le principal artisan de la reconnaissance universitaire de la discipline sociologique en France, Durkheim n'est pas pour autant le fondateur de la sociologie d'un point de vue intellectuel. Pour tempérer les anachronismes et les reconstructions héroïques du rôle des «pères fondateurs ", il nous semble essentiel de pouvoir replacer la construction durkheimienne dans l'histoire longue des analyses sociologiques du suicide. Certains aspects de l'œuvre de Durkheim sont alors éclairés d'un jour nouveau.

Ce n'est pas sans raison que, passant en revue la bibliographie comparée sur le suicide en Europe, Morselli estime en 1879 (506) que la littérature française sur les statistiques du suicide est la plus riche et la plus savante. Il est avéré que, en France, la collecte de données numériques sur le suicide commence au plus tard en 1817 , sous la responsabilité des médecins du Conseil de salubrité de Paris (Moléon, 1829). Elle se perfectionne ensuite sous la Restauration marquée notamment par la constitution du Compte général de l'administration de la justice criminelle (1827) et par la création des Annales d'Hygiène Publique et de Médecine Légale (1829), puis sous la monarchie de Juillet qui rétablit en 1833 le Bureau de statistique générale qui deviendra en 1840 la Statistique générale de la France (Lécuyer, 1977; Le Mée, 1979). Et dès la première moitié du XIX ${ }^{\mathrm{e}}$ siècle, certains statisticiens rai-

* CNRS, Centre de Recherches Sociologiques sur le Droit et les Institutions Pénales (CESDIP), Paris.

** Institut d'Histoire de la Médecine et de la Pharmacie de l'Université Paris V. 
sonnent bel et bien en termes de corrélations sociologiques. Ainsi, dès 1835, dans son Essai de physique sociale, Adolphe Quetelet traitait le suicide comme un fait social en insistant sur une effrayante concordance qui fait cette régularité dans un acte qui paraît si sensiblement lié à la volonté de l'homme (Quetelet, 1835, 414). Soixante-deux ans avant Durkheim, Quetelet écartait déjà l'individu pour s'intéresser au groupe dans son ensemble en postulant qu'il a en tant que groupe des tendances au suicide:

Nous devons, avant tout, perdre de vue l'homme pris isolément, et ne le considérer que comme une fraction de l'espèce. En le dépouillant de son individualité, nous éliminerons tout ce qui n'est qu'accidentel; et les particularités individuelles qui n'ont que peu ou point d'action sur la masse s'effaceront d'elles-mêmes, et permettront de saisir les résultats généraux (Quetelet, 1835, 31) ${ }^{1}$.

Les outils se précisant au fil des réformes de la statistique, au début des années 1880 - pour ne parler que des recherches françaises - le démographe Jacques Bertillon (18511922) peut établir à la suite de l'ouvrage du plus haut intérêt de Morselli les corrélations entre suicide et divorce, suicide et nombre d'enfants des couples mariés, suicide et pratique religieuse (y compris la distinction entre catholiques et protestants), dans une dimension comparatiste européenne (Bertillon, $1880 ; 1882$ ).

Loin d'innover dans ces recherches, Durkheim s'inscrit donc dans une longue tradition d'utilisation macrosociologique des statistiques qui raisonne sur des groupes pensés comme des totalités dotés de traits collectifs, [...] des réalités sui generis, différentes des individus, appelant des méthodes d'analyse spécifiques (Desrosières, 1993, 58, 96). En fait, la plupart des généralisations empiriques de Durkheim n'étaient autres que celles qui étaient issues de la statistique morale et qu'[il] avait reprises, précisées et surtout utilisées pour construire une théorie (Besnard, 1987, 154). Et c'est là l'essentiel. L'originalité de la démarche durkheimienne ne réside donc pas dans les traitements statistiques qui accompagnent les démonstrations et les réfutations, et c'est à tort que l'on présente parfois Durkheim comme l'instaurateur d'un rationalisme expérimental en sciences sociales (Berthelot, 1995). Ses analyses statistiques sont certes d'une ampleur et d'une systématicité supérieures à celles mises en œuvre avant lui, mais elles ne constituent pas une nouveauté véritable, seulement une étape appelée à être dépassée à son tour par la suite. C'est en réalité la construction théorique qui fait la véritable spécificité du Suicide de Durkheim. Et cette construction se trouve articulée notamment autour d'une conception particulière du rapport individu/ société, conception qui tranche singulièrement avec celle des psychiatres et des statisticiens qui ont précédé Durkheim, tout autant qu'avec celle d'Halbwachs trente ans plus tard.

\section{Durkheim, lecteur des théories psychiatriques}

Nonobstant sa polémique avec Tarde qu'il entend bien régler une fois pour toutes à cette occasion, en 1897 Durkheim se positionne dans un champ scientifique au sein duquel

\footnotetext{
On peut signaler aussi le texte «Du suicide et de ses causes » attribué (sans doute à tort) à un ancien haut fonctionnaire qui dirigea notamment la police parisienne pendant la Révolution. Ayant échappé à l'oubli parce qu'il fut traduit par Karl Marx en 1846, ce texte (qui, quoi qu'il en soit, date des années 1830) commençait par ces mots: Le chiffre annuel des suicides, en quelque façon normal et périodique chez nous, ne peut être considéré que comme le symptôme d'un vice constitutif de la société moderne, car à l'époque des disettes et dans les hivers rigoureux, ce symptôme est toujours plus manifeste, de même qu'il prend un caractère épidémique lors des haltes de l'industrie et quand les banqueroutes se succèdent en ricochet. La prostitution et le vol grandissent alors dans les mêmes proportions. Plus loin il écrivait aussi cette phrase que n'aurait pas reniée Durkheim: La classification des diverses causes de suicides serait la classification même des vices de la société (Peuchet, 1992, 33 et 60).
} 
ses principaux rivaux ne sont ni des sociologues, ni des anthropologues, mais avant tout des psychiatres ${ }^{2}$. Contre les théories qui voient dans le suicide la manifestation d'un trouble psychique individuel, Durkheim veut établir que le suicide est bien un fait social, une tendance collective qui dépend de l'état de la société et donc ressortit à la sociologie (Durkheim, 1897, 15-16). C'est du reste sur l'examen des thèses psychiatriques que s'ouvre son livre.

\section{La critique de l'approche médicale du suicide par Durkheim}

Partant du principe que les aliénistes voient dans toute mort volontaire une manifestation vésanique (Durkheim, 1897, 20), Durkheim en déduit que cet angle d'approche réduit l'étiologie du suicide à une affection individuelle. Nous reviendrons sur cette erreur, tant au niveau du constat que de la déduction, un peu plus loin. Le sociologue avance que la théorie de l'aliénation du suicidaire a été formulée sous deux formes. La première tend à considérer le suicide comme un symptôme nécessaire et suffisant pour forger une catégorie nosologique, la seconde voit dans le suicide la manifestation d'affections mentales variées. Durkheim objecte à cette dernière hypothèse de ne pouvoir être prouvée car il est impossible d'apprécier l'influence de l'aliénation mentale sur tous les cas de suicides et, quand même des exemples contraires ne seraient pas allégués, il y en aurait pourtant toujours de possibles (ibid., 21). C'était s'appuyer ici sur une critique radicale de l'inductivisme. D'une manière étrange pourtant, Durkheim ne suit pas le même raisonnement pour la thèse de folie spécifique. C'est la monomanie suicide qui serait la plus représentative de cette approche. Toutefois, il reconnaît immédiatement que cette théorie clinique est universellement abandonnée (ibid., 23) et il déduit de ce rejet que le suicide n'est pas une folie distincte (ibid., 26). Il ne reste plus alors à Durkheim qu'à discuter pour la forme la seconde hypothèse qu'il impute aux aliénistes, celle du suicide provoqué par des manifestations vésaniques non spécifiques. Pour analyser l'intérêt de cette relation causale, il juge qu'il convient d'abord de classer les suicides d'aliénés suivant les manifestations morbides dont ils relèvent, pour voir ensuite si ces formes permettent de rendre compte de tous les cas de suicides. Durkheim distingue alors les suicides maniaque, mélancolique, obsessif et impulsif ou automatique. Ces types ne lui semblent pas permettre d'expliquer tous les suicides car ils partagent la caractéristique d'être commis sans motifs ou sous l'emprise d'un raisonnement délirant sans fondement dans la réalité. Or, à la différence de l'aliéné, l'homme normal dépressif se tue toujours, selon Durkheim, pour une cause objective qui n'est pas sans aucun rapport avec les circonstances extérieures (ibid., 31). Ainsi, les aliénistes n'auraient pu maintenir cette seconde théorie explicative, tendant à voir en tout suicidaire un aliéné, qu'en excluant du champ du suicide tous les comportements suicidaires légitimés par la société. Il y a là un point de méthode que Durkheim ne peut accepter car cette attitude revient à définir le suicide en fonction de la nature des mobiles qui le guide. Si l'on rejette ce tri préalable, il lui paraît évident qu'il existe des suicides commis au nom d'une philosophie, d'une religion ou d'une cause patriotique, dans lesquels il n'entre aucun caractère de l'aliénation mentale: les mobiles ne sont pas fictifs et le suicidé a délibérément choisi de commettre son acte (ibid., 32-33).

Il reste à Durkheim, pour clore cette critique, à examiner ce qu'il considère comme un état intermédiaire entre l'aliénation et l'équilibre mental parfait: la neurasthénie.

2 Au demeurant sa critique de la théorie de l'imitation de Tarde n'est, du point de vue intellectuel, qu'un aspect particulier de sa critique des thèses psychiatriques qui utilisent les notions de contagion et d'imitation depuis plus d'un demi-siècle. 
Durkheim affirme que la neurasthénie prédispose au suicide et il juge que ce type psychologique est celui qui se rencontre le plus généralement chez les suicidés $(1897,35)$. Il ne doit pourtant pas être reconnu comme déterminant dans le passage à l'acte. Pour le prouver, Durkheim pose que le nombre de neurasthéniques varie avec celui des fous. Il lui suffit donc de montrer ensuite que le taux d'aliénés ne varie pas de manière concomitante avec celui des suicidés pour écarter l'hypothèse d'une relation causale entre la neurasthénie et le suicide (ibid., 33-46).

\section{Retour sur le débat médical}

Le fait que Durkheim débute sa revue critique des facteurs extra-sociaux par les théories des aliénistes n'est pas un hasard. Ce sont en effet les médecins et les aliénistes qui dominent le débat scientifique sur la question de l'étiologie du suicide depuis le début du $\mathrm{XIX}^{\mathbf{e}}$ siècle. Il faut donc effectuer maintenant un bref retour sur ce courant discuté par Durkheim. Dès le XVIII ${ }^{e}$ siècle en fait, certains ouvrages de médecins mettent directement en relation le suicide avec la folie d'une part, avec la mélancolie de l'autre. Hufeland définit la melancholia suicidum, Boissier de Sauvages et Balme décrivent la melancholia anglica, Pinel parle d'une mélancolie avec penchant au suicide, etc. Au-delà des différences nosologiques, les cliniciens s'accordaient pour affirmer que le suicide étant le résultat d'une maladie, c'était aux médecins d'en prévenir les effets (Chevrey, 1816, 7).

La métaphore médicale eut des contenus variés mais la revendication de compétence ne fut pas seulement théorique. Elle trouva très vite à s'exprimer auprès des autorités administratives à travers le Conseil de salubrité de Paris qui avait, entre autres charges, celle de tenir des statistiques de mortalité dans la capitale (Renneville, 1997a, 160ss.). Le rapport général d'activité de 1821 rappelle à ce sujet que les médecins du Conseil ont suggéré en vain et à plusieurs reprises aux préfets précédents d'autoriser une suite de recherches sur les suicidés que l'on dépose à la Morgue. L'objectif du Conseil de salubrité était de déterminer les causes qui poussent tant de malheureux à se défaire de la vie (Moléon, 1829, 203). En les connaissant, affirmait-il, l'administration pourrait agir en conséquence et prévenir ou diminuer le nombre de suicides. Dans le rapport d'activité de 1826, le Conseil dressait un premier tableau récapitulatif des suicides parisiens de 1817 à 1824 comprenant la capitale et les communes relevant de l'administration du préfet de Paris (soit Sèvres, Saint-Cloud et Meudon, situées en Seine-et-Oise). Ce tableau fait apparaître environ 350 tentatives par an en moyenne, $230 \mathrm{chez}$ les hommes et $120 \mathrm{chez}$ les femmes. Le rapport estime que ce chiffre est exhaustif car la tentative de suicide n'étant pas punie en France, le risque de cas dissimulés est négligeable. Surtout, le Conseil mettait déjà en évidence un phénomène que Quetelet allait révéler dans ses travaux de statistique morale: la relative régularité du chiffre d'une année sur l'autre, ainsi que la prédominance du sexe masculin (ibid., 306). La consultation des rapports successifs fait apparaître l'affinement progressif de l'analyse. Dans celui de l'année suivante par exemple, le Conseil de salubrité différencie non seulement les suicidés par sexe, mais aussi par situation familiale. On enregistre ainsi 218 suicides de célibataires, contre 176 d'individus mariés. Si les membres du Conseil pensaient qu'il y avait une faible oscillation du nombre de suicides d'une année sur l'autre, le rapport de 1826 les détrompa car il accusa 511 tentatives (et 357 réussites) contre 396 tentatives en 1825 et 371 en 1824 . En réaction, les médecins affinèrent leur analyse et ils donnèrent pour la première fois, dix ans avant le Compte général de l'administration de la justice criminelle, les motifs des suicides (déduits d'après les procès-verbaux de police). A la lecture de cette première distribution des motifs, un constat s'impose: les causes maladives $(30 \%)$ forment la catégorie 
la plus importante, suivie surtout par la passion (20\%) et la misère (20\%). L'un des problèmes de cette première répartition chiffrée est qu'elle mêle l'aliénation et les suicides consécutifs à des maladies incurables ou entraînant une grande douleur physique ou morale. Il est certain en tout cas que si le suicide est un sujet médical, il relève plus de l'hygiène que du traitement psychiatrique puisque les causes pathologiques expliqueraient moins d'un tiers des cas.

C'est à partir des années 1840 que les médecins discutèrent trois conceptions médicales du suicide. La première, celle d'Esquirol, faisait dépendre tous les suicides d'une aliénation non spécifique. La seconde, avancée par Bourdin, reliait tous les suicides à une maladie mentale spécifique: la monomanie suicide. La troisième enfin, qui l'emportera sous le Second Empire, partageait les suicides entre différentes causes, l'aliénation étant souvent reconnue comme la plus fréquente et parfois noyée au milieu d'un grand nombre de causes. Assez rapidement, les médecins tentèrent de pallier l'absence d'analyse détaillée des motifs de suicides en conjuguant données statistiques et analyses de dossiers d'instruction judiciaire. Gustave Etoc-Demazy, élève de Ferrus et médecin en chef de l'asile des aliénés de la Sarthe, publia en 1844 une enquête statistique localisée dans l'arrondissement du Mans, portant sur la période 1830 à 1841 inclus. L'aliéniste basa son interprétation étiologique sur le dépouillement des procès-verbaux et rapports rédigés par les officiers de police et les médecins légistes, déposés au parquet du tribunal de première instance. Etoc-Demazy rejetait l'hypothèse que tous les suicidés soient des aliénés, comme l'avait affirmé Esquirol, mais il pensait tout de même que les deux tiers des suicidés manifestaient un déséquilibre mental (Etoc-Demazy, 1844, 169-180). Cette conclusion fut approuvée par Alexandre Brierre de Boismont dans les Annales Médico-psychologiques, la principale revue de psychiatrie française de l'époque (Brierre de Boismont, 1844, 496). Elle fut toutefois à l'origine d'une polémique avec ClaudeEtienne Bourdin, qui publia l'année suivante un ouvrage très discuté tendant à assimiler tout suicide à une monomanie qui pouvait se répandre sous forme d'épidémie, comme on l'avait observé à Etampes et Versailles (1793), Rouen (1806), Saint-Pierre-Mont-Jean (1813) (Bourdin, 1845, 46-47). Bien qu'il défendit une monomanie, Bourdin n'appuyait pas sa démonstration sur la constance de lésions organiques. Mais le plus important pour notre propos est probablement là: si Bourdin médicalise totalement le suicide, il ne cherche pas sa première origine dans l'aliénation (il sait bien qu'il y a des aliénés qui ne se suicident pas) mais il distingue, comme ses prédécesseurs, un grand nombre de causes, qu'il regroupe en deux ordres. Il y a d'abord les causes prédisposantes, au premier rang desquelles se trouvent l'hérédité et l'imitation. Il y a ensuite les causes déterminantes et on retrouve ici les mêmes agents que dans la production de la folie: l'amour déçu, la haine et la jalousie, l'ambition trompée, les mécomptes de la fortune [...]. On pourrait ainsi passer en revue toutes les passions et trouver des exemples de suicides provoqués par chacune d'elles (ibid., 64). Le suicide et la folie relevant de la même étiologie, Bourdin proposait un arsenal thérapeutique similaire pour les deux affections. L'objectif de cette médicalisation du suicide était, comme pour la majorité des médecins, la déculpabilisation de l'acte:

Je dis que le suicide est toujours une maladie, et toujours un acte d'aliénation mentale: je dis par conséquent qu'il ne mérite ni louange ni blâme (Bourdin, 1845, 9).

Les aliénistes partageaient ainsi, au-delà de la querelle nosologique, une attitude paradoxale: d'un côté, ils revendiquaient la nécessaire déculpabilisation du suicide, de l'autre, ils reconnaissaient unanimement que l'étiologie du suicide et sa prévention touchait comme pour toute folie - à la fois à la médecine, à la morale et à la religion. 
Bourdin ne fut pas suivi dans son explication exclusive par la monomanie suicide. On a vu qu'Etoc-Demazy et Brierre de Boismont contestaient son interprétation. Le rejet fut presque unanime. Le 2 avril 1846 par exemple, la Société de médecine pratique discuta un mémoire de Bourdin, lu par le docteur Belhomme, qui en refusait les conclusions. Dans la discussion qui suivit, les docteurs Fouquier, Masson et Dancel critiquèrent également la position de Bourdin ${ }^{3}$. Etoc-Demazy répliqua encore dans les Annales Médico-psychologiques de la même année en affirmant que la mort volontaire n'est pas toujours et nécessairement un fait d'aliénation mentale (Etoc-Demazy, 1846, 362). Bourdin réagit à son tour dans la même revue en affirmant une nouvelle fois sa position, sans apporter d'éléments nouveaux (Bourdin, 1846a). Il publia également une réponse aux objections que Brierre de Boismont avaient formulées dans les Annales d'Hygiène Publique et de Médecine Légale. La controverse prit ici un tour plus argumenté. Bourdin refusait en particulier que la cause des suicides soit à rechercher, comme l'avait affirmé Brierre, dans le défaut d'éducation religieuse, la misère, les passions, les maladies, les vices ou les mauvaises habitudes. Il posait que son collègue confondait la cause avec le prétexte, la cause efficiente avec la cause déterminante:

Que l'on compare la fréquence des causes indiquées ci-dessus avec la rareté de l'acte (suicide) et l'on sera certainement convaincu qu'il n'existe pas un rapport intime entre le nombre incalculable des causes apparentes et le résultat qu'on leur attribue (Bourdin, $1846 \mathrm{~b}, 466)$.

Pour maintenir la monomanie suicide, Bourdin invoquait exactement l'argument mis au point par les aliénistes pour défendre la monomanie homicide devant les tribunaux: les aliénés suicidaires possédaient bien dans certains cas leur libre arbitre, mais jamais leur liberté morale (ibid., 467). Les symptômes de la folie suicide étaient donc à rechercher, chez les individus apparemment les plus lucides, dans les antécédents biographiques du malade, et non dans le seul fait de se donner la mort...

Alors que la monomanie homicide obtint un certain succès, la monomanie suicide ne fut jamais défendue aussi ouvertement que chez Bourdin. Même Louis Delasiauve, que Bourdin reconnaissait comme son maître, prit la peine de faire une mise au point en se désolidarisant de son élève (Delasiauve, 1850, 2).

Un autre signe fort de cette emprise des médecins sur la recherche des causes du suicide est donné par les thèses de médecine publiées sur ce thème tout au long du XIX ${ }^{e}$ siècle. S'il n'est pas question de les passer en revue ici, on peut retenir que les médecins se démarquaient de l'interprétation exclusive de Bourdin, le problème étant plutôt de connaître la proportion de suicides consécutifs à l'aliénation. Comme le notera justement J.-B. Petit dans une thèse parisienne de 1850 , déterminer la part de l'aliénation mentale dans la production du suicide est une des questions les plus épineuses et les plus vivement débattues de la pathologie mentale (Petit, 1850, 28-29). Les médecins avaient conscience qu'ils devaient résoudre ce problème en passant par le recueil de données chiffrées.

En 1856, l'aliéniste Alexandre Brierre de Boismont publia un ouvrage sur le suicide qui obtint suffisamment de succès pour être réédité neuf ans après. Brierre de Boismont admettait comme J.-B. Petit la possibilité de causes associées. L'originalité de son enquête résidait dans le dépouillement de 4595 procès-verbaux de suicides collationnés à la préfecture de police. Sur cette base, l'aliéniste distinguait, comme ses prédécesseurs, des causes prédisposantes et des causes déterminantes. Parmi les premières, on relève l'hérédité, le climat, le sexe, l'âge, l'état civil, l'état de fortune, la moralité, l'instruction, la pro-

3 Annales Médico-psychologiques, 1846, 8, 132. 
fession. Parmi les secondes, il y avait les passions et les causes variées, que l'aliéniste classait dans la deuxième édition de son ouvrage en dix groupes. Si l'on recompose cette distribution, trois catégories dominent: les causes économiques (pauvreté, misère, paresse, revers de fortune, manque d'ouvrage) représentant environ $20 \%$ des cas, les chagrins domestiques et sentimentaux pour environ $16 \%$ des cas, enfin les folies pour seulement $14 \%$ des cas.

En cette même année 1856, l'aliéniste Egiste Lisle publia un ouvrage sur le suicide qui se basait une nouvelle fois sur les chiffres donnés par le Compte général de la justice criminelle. Son angle d'approche dénotait toutefois un déplacement d'intérêt vers les causes "générales» du suicide ainsi qu'un tournant moralisateur et réprobateur qui annonçait l'œuvre de Durkheim ${ }^{4}$. Lisle n'entendait pas étudier en effet le suicide comme une maladie individuelle dont rien ne prou[vait] l'existence, mais comme un fait général malheureusement trop commun, et dénotant, au sein de nos sociétés modernes, en apparence si prospères, un malaise profond et caché qui les ronge jusque dans leurs éléments les plus sains (Lisle, 1856, V). Il souhaitait en chercher les lois générales et distinguait dans ce but, à la suite de ses prédécesseurs, les causes prédisposantes au suicide (climat, âge, sexe, profession, instruction...) et ses causes déterminantes (chagrins, passions, maladies, dont la folie). Lisle refusait également de voir dans le suicide un signe constant de folie. Pour ce médecin, c'était l'absence de lois répressives et le relâchement des mœurs, préparé par la philosophie des Lumières, qui étaient à l'origine de la croissance du suicide au XIX ${ }^{\mathrm{e}}$ siècle.

Durkheim s'intéressa aussi beaucoup à une enquête publiée par le docteur Emile Le Roy en 1870 sur le suicide en Seine-et-Marne. A la différence des études précédentes, Le Roy avait travaillé sur 2049 dossiers de procédure répartis dans le département tout entier et il comptait dans son échantillon un nombre important de suicides de population rurale. Pour ce médecin, les causes générales qui influent à la fois sur la répartition des maladies mentales et du suicide étaient avant tout des causes sociales (Le Roy, 1870, 226) mais toujours particulières: telle position familiale, tel changement de culture etc. Moyennant quelques recoupements, Le Roy $(1870,184-185)$ donnait la répartition suivante: maladies mentales (38\%), accusés (justiciables) $(24 \%)$, misère ( $21 \%)$, ivresse $(6 \%)$, dégout et souffrance $(6 \%)$. Il refusait l'existence d'une folie suicide spécifique. Si l'on compare cette classification avec celle de Brierre de Boismont, on constate immédiatement que les catégories ne sont pas homogènes. Le point commun des deux auteurs réside ici, contrairement à ce qu'affirmera Durkheim, dans la volonté de donner une portée sociale au suicide. Le Roy l'affirme très clairement:

Le suicide est donc un reflet des tendances de l'époque et toutes les fois qu'il se produit épidémiquement, il indique ordinairement un état de gêne intime de la société. On le voit, en effet, suivre toutes les vicissitudes de la vie humaine, augmentant aux époques d'isolement, de découragement et de décadence, avec le défaut absolu de croyance et le scepticisme en ce qui concerne les devoirs envers le pays et la société $(1870,253-254)$.

En 1881 encore, Legoyt publie une recherche sur le suicide utilisée par Durkheim qui distingue très nettement les causes individuelles et les causes générales du passage à l'acte. Cet ancien chef des travaux de la statistique de la France juge déjà que les causes générales du suicide exercent sur les causes individuelles une influence irrésistible, bien qu'invisible (Legoyt, 1881, 295). Legoyt décrit même l'accroissement du taux de suicide en des termes que n'auraient pas reniés Durkheim car le phénomène ne peut s'expliquer selon lui que

4 Ce tournant est plus évident encore chez le docteur Ebrard qui rappelle en 1870 que le suicide est un crime face à la théologie (Ebrard, 1870,1-3). Quant à Durkheim, il légitimera très clairement la condamnation sociale du suicide $(1897,376-384)$. 
par des causes générales, dont l'individu qui se tue subit l'effet plus ou moins à son insu (ibid., 297). L'explication diffère essentiellement par le contenu des causes générales. Pour Legoyt, il faut compter avec l'augmentation de la pauvreté, la crise économique (ibid., 298-306), l'affaiblissement du sentiment religieux, reconnu par tous les physiologistes sans exception, comme le préservatif le plus efficace contre le suicide (ibid., 308), la perte graduelle du sentiment de la famille (ibid., 312), l'affaiblissement enfin de l'esprit de concorde et de charité (ibid., 328).

Il n'est pas utile pour notre propos de poursuivre ainsi la revue des publications à caractère médical sur le suicide durant les années 1880 et 1890 . Les thèses de médecine passées sur ce thème sont en effet redondantes et si la théorie de la dégénérescence tend à s'imposer comme modèle psychopathologique face à la clinique des monomanies, la théorie du suicide considéré systématiquement comme un symptôme de folie n'est plus défendue après Bourdin. Albert Tibéri encore, en 1895, notera dans une thèse lyonnaise que le suicide peut parfaitement exister en dehors de la folie. Il reconnaît alors des causes morales ou sociales qui sont de la compétence des philosophes et des sociologues (Tibéri, $1895,6)$.

La seule innovation conceptuelle notable de cette fin de siècle est, pour l'œuvre durkheimienne, le succès de la notion de neurasthénie. Présent dans l'œuvre de Durkheim dès 1893 dans la Division du travail, ce terme se retrouve également à l'époque sous la plume d'écrivains comme Octave Mirbeau, Jules Renard et Paul Valéry (Bablon-Dubreuil, 1996). Ce qu'il faut en retenir simplement, c'est que le concept est d'origine médicale. Il a été proposé par George M. Beard en 1869 pour décrire un état d'épuisement nerveux de plus en plus fréquent dans les pays civilisés (Beard, 1869). Peu discutée dans un premier temps aux Etats-Unis, la neurasthénie est en revanche rapidement diffusée en Europe à partir des années 1880. En France, l'aliéniste Joseph Grasset l'utilise et Jean Charcot l'impose à la communauté médicale dans les cours de clinique qu'il donne à La Salpêtrière (Charcot, 1890-1892, vol. I). Charcot décrit avec minutie en particulier la série des stigmates neurasthéniques: céphalées, insomnie, dépression cérébrale, asthénie neuro-musculaire, rachialgie et dyspepsie par atonie gastro-intestinale. Dans les années 1890, le terme de neurasthénie devient à la pathologie nerveuse ce que la dégénérescence est à la maladie mentale: un syndrome fédérateur, un creuset conceptuel qui recouvre de nombreuses descriptions autrefois distinguées. En fait, bien des médecins admettent que la précision du terme est inversement proportionnelle au champ potentiel qu'on assigne à sa valeur diagnostique (par ex. Bouveret, 1891, 6).

Deux remarques s'imposent sur cette question. La première, c'est que l'invocation de la neurasthénie comme cause de suicide était marginale dans le discours médical car les médecins n'y voyaient le plus souvent qu'un syndrome bénin dont il était possible de faire disparaître les effets par du repos, des bains ou de l'électricité. La seconde remarque concerne le statut du concept de neurasthénie dans l'œuvre de Durkheim. Rappelons d'abord ici que Durkheim se considérait lui-même comme un neurasthénique, ce qui aide à comprendre la description très empathique et non critique qu'il fait de cette source éminemment féconde de nouveauté dans son étude sur le suicide $(1897,45)^{5}$. Toutefois, si Durkheim s'accorde à penser avec les médecins que cette maladie progresse dans la société, il juge que cette progression n'a rien d'anormal car c'est au moment précis où les neurasthéniques sont les plus nombreux qu'ils ont le plus de raisons d'être $(1897,45)$. Un principe de Pangloss que Durkheim n'appliquait pas à l'accroissement du taux de suicide qui était, lui, pathologique, comme nous le verrons plus loin.

s Sur la neurasthénie de Durkheim telle qu'elle se dévoile dans sa correspondance, $c f$. Mucchielli (1998b). 


\section{Les biais de Durkheim}

Bien que la revue des travaux de psychiatrie proposée ci-dessus soit rapide et parcellaire, elle nous permet de mieux apprécier la lecture de Durkheim. Sachant qu'il n'entre pas dans notre dessein de défendre ici telle ou telle explication causale, on peut remarquer que Durkheim fait une lecture du corpus psychiatrique qui est guidée par plusieurs réductions. Il faut noter d'abord qu'au moment de la publication du livre de Durkheim, c'est la dégénérescence qui structure la psychopathologie. Durkheim accuse ici un décalage certain avec le savoir psychiatrique de son époque. Plutôt que de discuter la théorie de la dégénérescence, qu'il semble par ailleurs cautionner au moins partiellement par l'usage du terme de dégénéré $(1897,46,53)$, il convoque pour sa critique la clinique des monomanies $^{6}$. Il est évident que ce tri lui facilite la tâche car il y a alors déjà un peu plus de quarante ans que les aliénistes ont entrepris de critiquer cette nosologie et au moment où Durkheim la discute, elle est tombée en désuétude depuis plusieurs décennies. Comment expliquer ce choix qu'il ne justifie pas?

Il existe un problème plus fondamental encore, qui réside dans la typologie des causes proposée par Durkheim. Si les aliénistes s'étaient intéressés à l'ouvrage du sociologue (ce qui ne semble pas être le cas), on peut gager que ceux-ci auraient certainement trouvé bien étrange le fait que les dispositions organico-psychiques en général et les causes psychiques en particulier soient classées parmi les facteurs «extra-sociaux». Ceci pour deux raisons essentielles. D'abord, le rapport entre l'extension de la folie et les milieux sociaux était l'une des questions au cœur de la psychiatrie de l'époque et l'on se demanda, très tôt dans le siècle, si la civilisation ou le développement des villes favorisait les pathologies mentales. Ensuite, et surtout, la théorie de l'hérédité des caractères acquis, alors admise par les médecins français, permettait sans contradiction théorique de relier les pathologies et les milieux sociaux. Durkheim n'ignorait d'ailleurs pas ce point, puisqu'il écrivait dès 1888 , à propos des origines du suicide que les causes organiques ne sont souvent que des causes sociales transformées et fixées dans l'organisme (Durkheim, 1888, 462).

L'autre réduction que Durkheim fait implicitement, c'est d'affirmer que les psychiatres médicalisent tous les suicides. Ceci est faux, nous l'avons vu plus haut. Et Durkheim, qui connaît bien la littérature psychiatrique sur le suicide, ne peut soutenir cette hypothèse qu'en faisant un usage tronqué des citations. Nous n'en donnerons qu'un exemple. Lorsqu'il cite Brierre de Boismont comme auteur accréditant la monomanie suicide comme cause des suicides, il oublie de préciser que le suicide n'est pas toujours pour cet aliéniste une preuve de folie. Si le médecin affirme en effet que le nombre des monomanies suicide et homicide est réellement considérable (Brierre de Boismont, 1856, 140) comme le relève Durkheim $(1897,22)$, le sociologue oublie de préciser que ce considérable ne renvoie qu'à un septième des cas analysés. Retournons ici aux chiffres de Brierre de Boismont que Durkheim ne cite pas: l'aliéniste décompte 652 cas de suicide par folie sur un total de 4595 dossiers (soit seulement 14\%). Et parmi ces 652 dossiers, il n'admet que 131 cas de monomanies (soit $2,85 \%$ du total). Les autres sont des folies liées à des craintes des ennemis, peur de la police, folie consécutive à un chagrin, folies consécutives à des maladies, dues à des idées diverses, la nostalgie, l'épilepsie, la démence, la masturbation et l'intoxication au blanc de céruse, etc.

- Sur l'influence de la pensée médicale dans les premiers textes de Durkheim, on se reportera aussi à l'article de R. Nye (1982) qui tend toutefois, à notre sens, à en exagérer la portée dans l'ensemble de l'œuvre durkheimienne. 
La divergence d'approche entre Durkheim et les aliénistes réside plus profondément dans la conception même de la folie. Alors que le sociologue accorde à celle-ci le statut d'une cause hypothétique, les aliénistes considèrent la pathologie mentale comme un ensemble de symptômes dont il faut rechercher l'étiologie. Pour employer la terminologie de Thomas Kuhn, on peut dire qu'il y a dans cette différence d'approche une incommensurabilité épistémologique: Durkheim discute la folie comme une cause improbable mais finale de suicide tandis que les psychiatres y voient la conséquence médiante de causes organiques et/ou morales, voire sociales. Faire coïncider ainsi une pathologie mentale à une causalité individuelle et organique participait déjà, à l'époque, d'une conception réductrice du champ de la pathologie mentale. Durkheim base en effet son raisonnement sur le postulat que l'assignation par les médecins d'un terrain pathologique au suicide implique que ce dernier ne soit qu'une affection individuelle $(1897,20)$. Or nous avons vu que bien peu de médecins étaient prêts à souscrire à une relation aussi étroite et exclusive, même chez les tenants de la monomanie suicide. La pensée médicale du XIX ${ }^{e}$ siècle ne distinguait donc pas aussi nettement l'individuel et le social.

Retenons l'essentiel de cette première partie. Durkheim montre par ses références qu'il connaît bien la littérature psychiatrique de son sujet. Comment expliquer alors ses erreurs d'interprétation? Pourquoi ne rend-il pas compte de toutes les nuances du positionnement des médecins? Pourquoi ne joue-t-il pas plus sur leurs propres contradictions? L'historien ne peut proposer ici que des hypothèses. Il est clair par exemple que bon nombre d'aliénistes ont médicalisé certains suicides dans l'intention de déculpabiliser l'acte; ce que ne pouvait que contredire un Durkheim qui posait à la fin de son étude qu'il était nécessaire que le suicide soit classé au nombre des actes immoraux (Durkheim, 1897, 383). En plein accord ici avec Berrios et Mohanna (1990), il nous semble que la lecture de Durkheim ressortit d'une stratégie d'institutionnalisation. Sa lecture est d'autant plus biaisée qu'elle doit mettre en valeur la nouveauté de ses propres analyses. Durkheim a même dâ écarter le concept de neurasthénie qui représentait certes un pont conceptuel possible entre l'individuel et le social, mais qui relevait trop ouvertement à l'époque de la compétence des médecins. Reconnaissant simplement que les neurasthéniques étaient des individus prédisposés au suicide en général, il s'est engagé dans une tout autre construction théorique.

\section{Le modèle durkheimien d'explication du suicide}

En 1897, Durkheim prétend instaurer une méthodologie diamétralement opposée à l'approche psychiatrique. Au lieu de partir de l'analyse des motifs présumés du suicide, appuyée ou non par des études sur dossiers, le sociologue annonce d'emblée qu'il ne s'intéresse qu'au taux de suicide et que son analyse statistique l'a conduit à mettre en évidence l'existence de plusieurs catégories de suicides qui correspondraient nécessairement à autant d'attitudes individuelles différentes. Notre éclairage historique portera ici sur deux points. Nous montrerons que cette position repose sur une disqualification de l'analyse des motifs au cours de laquelle Durkheim opère (volontairement ou non?) quelques «arrangements » avec les statistiques de l'époque. Nous verrons ensuite qu'elle s'appuie sur une théorie des mécanismes sociaux inconscients qui traduit une inflexion importante de la conception du rapport individu/société par rapport aux Règles de la méthode sociologique et qui, pour être bien comprise, doit être replacée dans le contexte particulier de la psychologie des années 1880-1890. 


\section{Le rejet de l'analyse des motifs présumés de suicides}

Ayant écarté dans le Livre premier du Suicide ce qu'il nomme les facteurs extra-sociaux (folie, race, hérédité, climat, imitation), Durkheim met en place son modèle d'explication. Celui-ci repose, on le sait, sur la typologie des trois (en fait quatre) suicides (égoïste, altruiste, anomique et, de façon marginale, fataliste). Mais le tout premier chapitre du Livre II est consacré à la disqualification de la statistique des motifs sur laquelle les psychiatres fondaient depuis un demi-siècle la plupart de leurs interprétations. Ce chapitre est donc à lui seul une nouveauté dans l'histoire des analyses du suicide et il conviendrait de l'examiner avec la plus grande attention. Or un tel examen est généralement absent des maruucls et des présentations de l'œuvrc de Durkhcim. Le problème de l'historien n'est pas ici de juger si Durkheim avait tort ou raison: au lieu de considérer ce point acquis au regard de la science actuelle, nous proposons de l'éclairer par les données utilisables à l'époque.

Durkheim affirme que, pour atteindre les causes du suicide, il n'est pas nécessaire d'examiner les motifs des gens qui se suicident. Pourtant, contrairement à une absence que regrettait jadis Quetelet $(1835,417)$, les recueils statistiques annuels de la France dont Durkheim dispose par exemple pour les années 1870-1890 contiennent une liste précise et constante des motifs présumés des suicides (chagrins de famille, misère et revers de fortune, maladie, amour contrarié, etc.). S'appuyant sur une remarque de Wagner sur la statistique allemande en 1864, Durkheim dénie globalement toute valeur à ces statistiques des motifs: confectionnées par des fonctionnaires s'autorisant des jugements improvisés sur la base de quelques renseignements hâtivement recueillis, il s'agit surtout d'une statistique des opinions que se font de ces motifs les agents, souvent subalternes, chargés de ce service d'informations (Durkheim, 1897, 144). Cet argument n'est pas nouveau: depuis Guerry et Quetelet en 1835, tous les statisticiens n'ont cessé de dénoncer le manque de formation des fonctionnaires de police chargés de l'enregistrement des suicides et ce doute sera une constante de la sociologie du suicide ${ }^{7}$. Mais le plus intéressant ici est le fait que Durkheim ajoute immédiatement:

Il y a plus, alors même que ces informations seraient dignes de foi, elles ne pourraient pas nous rendre de grands services, car les mobiles qui sont ainsi, à tort ou à raison, attribués aux suicides, n'en sont pas les causes véritables (ibid., 144).

Examinons les deux arguments qu'il avance.

Tout d'abord, tandis que le suicide a globalement augmenté de $40 \%$ en France entre 1856 et 1878, chaque catégorie de motifs conserve d'une époque à l'autre la même importance respective (ibid., 145). L'argument est étonnant car les statisticiens ont généralement soutenu le contraire: la constance serait plutôt une preuve de la validité. Quoi qu'il en soit, Durkheim entend prouver le bien-fondé de son idée à l'aide du Tableau XVII qu'il emprunte à Legoyt $(1881,342)$ et qui montre en effet une constance des motifs entre deux courtes périodes historiques distantes d'une quinzaine d'années: 1856-60 et 1874-78. Mais Durkheim n'a pas choisi ces dates au hasard. En effet, il aurait tout aussi bien pu reproduire le tableau de Morselli $(1879,397)$ (établi à partir de la même source), qui découpe

7 En 1967, c'était encore un argument central pour Douglas. Comme le faisait remarquer $\mathrm{Ph}$. Besnard $(1976,318)$, il est frappant cependant de constater que, de Durkheim à Douglas et Baechler, les utilisateurs de cet argument se sont bien gardés d'entreprendre des enquêtes précises sur les conditions d'enregistrement des décès. En somme, l'argument sert le contradicteur dans le débat des idées, mais ne fait pas progresser la recherche (inversement, $c f$. les éléments donnés par Chesnais [1976], ainsi que la mise au point pour l'enregistrement actuel in Baudelot et Establet [1984]). 
autrement la durée historique et indique au contraire que l'importance relative des différents motifs connaît bel et bien des changements importants (jusqu'à 26\%) entre 18511860 et 1861-1875. On vérifie ici ce fait bien connu qu'il suffit de peu de manipulation pour faire dire aux chiffres ce que l'on souhaite.

Allons plus loin. Non seulement Durkheim trie les données qu'il utilise, mais en empruntant ici et là quelques données souvent anciennes il néglige sciemment l'existence d'une source statistique constante permettant de mesurer annuellement l'évolution dans le temps des causes présumées de suicide. Il s'agit des données du Compte général de l'administration de la justice criminelle, reprises uniformément depuis 1878 dans l'Annuaire statistique de la France que publie chaque année le Service de la Statistique Générale de la France. Or, en dépouillant ces recueils, il est facile de reconstituer des séries permettant d'observer l'évolution des mobiles présumés de suicides sur longue période ${ }^{8}$. Durkheim y aurait constaté que cette distribution n'est ni statique, ni erratique, mais indique des tendances de moyen terme. A causes inconnues constantes et compte tenu de la croissance brute générale du nombre de suicides (d'environ 4500 à 7000 sur la période), on observe par exemple un doublement des suicides pour cause de souffrance physique entre 1876 et 1894 et des suicides pour causes de misère ou de ruine à partir de 1882 (début de la crise économique).

Le second argument de Durkheim est plus déroutant encore: la répartition des différents motifs se maintiendrait à peu près constante dans les diverses professions. Le tableau qu'il emprunte à nouveau à Legoyt montre en effet qu'à l'exception de l'ivresse, les agriculteurs et les professions libérales se suicident grosso modo pour les mêmes raisons et dans des proportions similaires (Durkheim, 1897, 145). Durkheim tire de ce constat une preuve de l'insignifiance des motifs présumés du suicide. Son argumentation est la suivante:

La vie d'un artiste, d'un savant, d'un avocat, d'un officier, d'un magistrat ne ressemble en rien à celle d'un agriculteur. On peut donc regarder comme certain que les causes sociales du suicide ne sont pas les mêmes pour les uns et pour les autres (1897, $145)^{9}$.

Durkheim s'appuie ici sur un pur postulat au terme duquel les types sociologiques du suicide correspondraient à des populations différentes. Ce glissement déductif est nouveau dans son analyse, il arrive soudainement et sans être étayé.

De plus, Durkheim ne produit ici qu'un tableau lacunaire lors même que la statistique criminelle fournit une distribution bien plus complète des motifs présumés des suicides selon les différentes catégories socioprofessionnelles (pour employer la terminologie moderne), distribution qui invalide son analyse. C'est ce que montre le Tableau I que nous avons reconstitué à la manière de celui que présente Durkheim $(1897,147)$ pour la même date. On y constate que, si les motifs des agriculteurs et des professions libérales présentent effectivement de grandes similitudes (sauf vis-à-vis de la souffrance physique qui inciterait les professions libérales au suicide plus souvent que les agriculteurs), il n'en va pas de même pour ceux des ouvriers, des artisans-commerçants ou encore des domestiques. Tandis que ces derniers se distinguent de l'ensemble des autres professions par une impor-

8 Ne pouvant entrer ici trop dans le détail, nous nous sommes contentés d'effectuer quelques sondages d'où sont extraites les informations qui suivent.

$9 \quad$ Son vocabulaire est révélateur: ce sont des forces très différentes qui poussent au suicide le laboureur et le raffiné des villes (ibid., 146; nous soulignons). N'est-ce pas la représentation du sauvage et du civilisé, banale au XIX ${ }^{c}$ siècle, qui sous-tend cette formulation? 
tance inédite des suicides pour amour contrarié et jalousie, les ouvriers et les artisans-commerçants semblent beaucoup plus sensibles à la misère ou à la ruine, plus enclins à se suicider sous le coup de l'ivresse et moins sous celui de la folie ${ }^{10}$.

Tableau I: Distribution des motifs présumés de suicide dans les différentes catégories socioprofessionnelles en 1878 (en \%).

\begin{tabular}{|l|c|c|c|c|c|}
\hline & Agriculteurs & Ouvriers & $\begin{array}{c}\text { Artisans - } \\
\text { commerçants }\end{array}$ & Domestiques & $\begin{array}{c}\text { Professions } \\
\text { libérales }\end{array}$ \\
\hline $\begin{array}{l}\text { Misère ou revers de } \\
\text { fortune }\end{array}$ & 6,7 & 11,2 & 13,2 & 7 & 8,9 \\
\hline Chagrin de famille & 12,8 & 12,8 & 14,2 & 10,7 & 12,8 \\
\hline $\begin{array}{l}\text { Amour contrarié et } \\
\text { jalousie }\end{array}$ & 2,8 & 1,9 & 3,3 & 11,7 & 2,8 \\
\hline Ivresse et ivrognerie & 13 & 21,1 & 16,7 & 13,7 & 7 \\
\hline Suicide d'accusés & 3,6 & 4 & 3,9 & 3,3 & 4,7 \\
\hline Souffrance physique & 15,7 & 13 & 13,5 & 12 & 20,7 \\
\hline Maladie mentale & 37,2 & 27,4 & 25,2 & 33 & 34,7 \\
\hline Dégoût de la vie & 3,1 & 2,7 & 2,6 & 4,3 & 4,6 \\
\hline Cause inconnue & 5,1 & 5,9 & 7,4 & 4,3 & 3,8 \\
\hline & 100 & 100 & 100 & 100 & 100 \\
\hline
\end{tabular}

Source: Annuaire statistique de la France (nous calculons les pourcentages).

Sans nous prononcer sur l'interprétation de ces statistiques, ce bref rappel des données disponibles à l'époque fait surgir, pour l'histoire de la sociologie, une question inédite: pourquoi et surtout comment Durkheim en est-il venu à poser une différence radicale entre l'observation des cas individuels et l'observation des tendances collectives? Que les deux méthodes soient différentes et que seule la seconde permette de mesurer le suicide comme phénomène social, cela va de soi. La difficulté est ailleurs. Si les causes du suicide sont, dans la très grande majorité des cas, avant tout sociales, alors on ne voit pas pourquoi il serait impossible aux sociologues d'en observer les effets sur les individus. Si on ne peut pas faire d'un seul cas individuel une généralité, on doit pouvoir observer une généralité sur certains cas individuels. Or c'est précisément ce que Durkheim nie catégoriquement:

Ce n'est pas une description, même bien faite, des cas particuliers qui pourra jamais nous apprendre quels sont ceux qui ont un caractère sociologique $(1897,143)$.

10 Ces catégories populaires se trouvent du reste globalement absentes des analyses de Durkheim. Dans son chapitre sur le suicide anomique, au terme de développements purement normatifs sur la valeur morale de la pauvreté et sur la nécessité de freiner ou contenir les besoins humains illimités, Durkheim va jusqu'à écrire cette contre-vérité étonnante (au regard des statistiques dont il disposait) que la pauvreté protège contre le suicide (ibid., 282). Nous ne pouvons donc que souscrire au jugement de J.-C. Chesnais (1981, 244): au XIX' siècle, c'est aux deux extrémités de la pyramide sociale que le suicide est le plus répandu. Quand Durkheim note que le suicide est exceptionnellement fréquent dans les classes les plus élevées de la société, il s'appuie sur un univers tronqué; il ne prend en compte que certaines des données [utilisables à l'époque]. Il abandonne le bas de la hiérarchie sociale [...]. Il ne mentionne pas le sort des miséreux. 
Et, à l'appui de cette affirmation visant à discréditer les études menées sur des grands nombres de cas par les psychiatres comme Brierre de Boismont, Durkheim met en avant l'inconscience des acteurs - les confidences que le sujet lui-même nous fait sur son état sont le plus souvent insuffisantes, quand elles ne sont pas suspectes. Il n'est que trop porté à se tromper sur lui-même et sur la nature de ses dispositions (ibid., 140-141). L'argument lui semble décisif car il découle directement de sa conception du rapport individu/société. C'est du moins ce que nous allons nous efforcer de montrer en suivant attentivement une évolution importante et pourtant rarement aperçue de Durkheim entre les Règles et le Suicide.

\section{L'individu comme condition et non cause du fait social}

Si le Suicide de Durkheim est considéré comme un classique de la sociologie et est enseigné comme tel aux étudiants, c'est qu'il est présenté comme, sinon la démonstration de la validité, du moins l'application des positions méthodologiques exprimées deux ans plus tôt dans les Règles de la méthode sociologique. De fait, c'est bien sur une mise en œuvre systématique de la méthode des variations concomitantes que se fonde en permanence Durkheim, aussi bien pour réfuter les hypothèses de ses adversaires que pour établir le bien-fondé des siennes. On sait qu'en effet, à ses yeux, cette méthode atteint le rapport causal, non du dehors, mais par le dedans,[...] elle nous montre deux faits participant l'un de l'autre et d'une manière continue, du moins pour ce qui regarde leur quantité. Or cette participation, à elle seule, suffit à démontrer qu'ils ne sont pas étrangers l'un à l'autre (Durkheim, 1947, 129). Sans doute, le rapport causal ainsi découvert n'est pas forcément direct (les deux phénomènes variant concomitamment peuvent par exemple être tous les deux sous la dépendance d'une même cause qui renvoie à un troisième phénomène), mais c'est assurément un moyen à la fois de contre preuve (l'absence de toute concomitance écarte la présomption de relation causale) et de preuve indirecte. Plus précisément encore, Durkheim se fonde sur le postulat qu'à un même effet correspond toujours une même cause, donc que la pluralité de causes constatées est la conséquence d'une mauvaise définition de l'objet qui doit être subdivisé. Dès 1894 (date de la rédaction des Règles), il écrit du reste que si le suicide dépend de plus d'une cause, c'est que, en réalité, il y a plusieurs espèces de suicides (ibid., 127-128). On devine qu'il a déjà commencé à écrire le Suicide.

Ceci est bien connu. Mais ce qui l'est moins, c'est la façon dont Durkheim ajoute à ce moment que, pour parvenir à une démonstration complète, l'explication sociologique doit rendre compte non seulement de la cause sociale d'un phénomène, mais encore de la conformité de cette cause avec ce que l'on pourrait appeler les données constantes de la nature humaine. Comme jadis Auguste Comte, Durkheim estime qu'une explication sociologique se vérifie in fine par sa vraisemblance du point de vue psychologique. L'exemple qu'il choisit dans les Règles est éloquent puisqu'il s'agit encore du suicide:

On peut établir de la manière la plus certaine que la tendance au suicide varie comme la tendance à l'instruction. Mais il est impossible de comprendre comment l'instruction peut mener au suicide; une telle explication est en contradiction avec les lois de la psychologie. L'instruction, surtout réduite aux connaissances élémentaires, n'atteint que les régions les plus superficielles de la conscience; au contraire l'instinct de conservation est une de nos tendances fondamentales. Il ne saurait donc être sensiblement affecté par un phénomène aussi éloigné et d'un aussi faible retentissement. On en vient ainsi à se demander si l'un et l'autre ne seraient pas la conséquence d'un même état. Cette cause 
commune, c'est l'affaiblissement du traditionalisme religieux qui renforce à la fois le besoin de savoir et le penchant au suicide (ibid., 131; nous soulignons).

Ce raisonnement peut surprendre de prime abord. Celui qui, dans le même ouvrage, affirme qu'il faut toujours expliquer le social par le social et écarte les raisonnements naturalistes comme des prénotions, invoque à son tour des instincts, des besoins, des tendances fondamentales de notre nature. Ces tendances sont incapables de générer à elles seules un comportement social; mais, parce qu'elles orientent l'activité de la conscience, elles auraient le pouvoir de diminuer ou au contraire d'accroître l'influence d'un phénomène social sur le comportement. Durkheim reconnaît donc implicitement qu'aucun fait social ne se produit mécaniquement, que les consciences individuelles sont le lieu d'une rencontre entre des contenants psychologiques très généraux et des contenus sociaux précis, les premiers déterminant l'efficacité des seconds. De ce que Durkheim a posé pour règle que la cause déterminante d'un fait social doit être cherchée parmi les faits sociaux antécédents, et non parmi les états de la conscience individuelle, on déduit généralement que l'individu ne joue plus aucun rôle dans son analyse, ce qui est donc inexact dans la lettre des Règles. Certes Durkheim refuse catégoriquement ce qu'il appelle le finalisme psychologique, c'est-à-dire le fait que l'on déduise les faits sociaux des données de la nature humaine générale (ibid., 97ss.). Mais si elles ne peuvent être des causes de faits sociaux, ces données en demeurent des conditions:

On se méprendrait étrangement sur notre pensée si, de ce qui précède [le rejet de la psychophysiologie individuelle], on tirait cette conclusion que la sociologie, suivant nous, doit ou même peut faire abstraction de l'homme et de ses facultés. Il est clair, au contraire, que les caractères généraux de la nature humaine entrent dans le travail d'élaboration d'où résulte la vie sociale. Seulement ce n'est pas eux qui la suscitent ni qui lui donnent sa forme spéciale; ils ne font que la rendre possible. Les représentations, les émotions, les tendances collectives n'ont pas pour causes génératrices certains états de la conscience des particuliers, mais les conditions où se trouve le corps social dans son ensemble (ibid., 105).

Ce n'est du reste pas la première fois que le sociologue raisonne de la sorte, et l'un des précédents les plus explicites se trouve dans son premier article sur le suicide. En 1888, tandis qu'il discute des rapports entre le suicide et la natalité, Durkheim en vient à montrer selon le même principe que les deux phénomènes dépendent d'une même cause:

Si le suicide progresse quand la natalité décline, c'est que ces deux phénomènes également sont dus en partie à une régression des sentiments domestiques (Durkheim, 1888, 462).

Et à nouveau, Durkheim entend prouver la validité de cette relation par sa conformité à la nature humaine:

D'où vient cette propriété bienfaisante de la famille? [...] Il n'y a qu'une réponse possible à la question: c'est que la vie en famille est dans la nature de l'organisme humain, tel du moins que l'a fait l'évolution (ibid., 463).

Et vingt ans plus tard, il écrira encore:

D'une manière générale, nous estimons que le sociologue ne s'est pas complètement acquitté de sa tâche tant qu'il n'est pas descendu dans le for intérieur des individus afin de rattacher les institutions dont il rend compte à leurs conditions psychologiques (Durkheim, 1909, 755; nous soulignons). 
Rattacher les causes sociales aux conditions psychologiques individuelles est donc un principe méthodologique récurrent chez Durkheim. Mais cette constance ne doit pas masquer que sa conception du rapport entre les deux ordres de réalités a pris un virage très nettement déterministe dans le Suicide, dans le sens d'une considération de plus en plus exclusive accordée aux facteurs sociaux externes censés agir directement et sans relais sur les consciences individuelles.

\section{Le tournant du Suicide: la causalité univoque du rapport individu-société}

Il faut insister sur ce point essentiel: dans l'introduction de son ouvrage, Durkheim rejette catégoriquement l'approche du suicide par les mobiles des individus. Il écrit d'emblée que la conscience ne peut pas connaître les raisons qui la déterminent:

L'intention est chose trop intime pour pouvoir être atteinte du dehors autrement que par de grossières approximations. Elle se dérobe même à l'observation intérieure. Que de fois nous nous méprenons sur les raisons véritables qui nous font agir! Sans cesse, nous expliquons par des passions généreuses ou des considérations élevées des démarches que nous ont inspiré de petits sentiments ou une aveugle routine (Durkheim, 1897, 4).

C'est donc une théorie des mécanismes inconscients du suicide que va construire Durkheim, estimant que les délibérations humaines, telles que les atteint la conscience réfléchie, ne sont souvent que de pure forme et n'ont d'autre objet que de corroborer une résolution déjà prise pour des raisons que la conscience ne connaît pas (ibid., 333-334). L'individu sait qu'il se tue, mais ne sait pourquoi, ou du moins se trompe sur les raisons véritables qui le font agir. C'est cette négation, suivie de la prétention de parvenir à identifier ces raisons véritables et à bâtir en somme une nouvelle théorie psychologique par la seule analyse de l'état de la société, qui font la singularité radicale de Durkheim à son époque. S'il s'était contenté de tester les relations entre suicide et famille, suicide et religion, suicide et crises économiques, suicide et révolutions politiques, et de mettre ainsi en évidence le poids global des conditions sociales dans l'importance et les évolutions historiques du taux de suicide, il se serait épargné bien des critiques, depuis celle de son propre collaborateur Simiand (1898) jusqu'à celles plus récentes des sociologues se réclamant d'un paradigme individualiste à la manière de Baechler (1975) qui raillait les considérations puériles et comiques de Durkheim, notamment sur les courants suicidogènes. Pourtant, loin d'être anecdotiques, ces passages du Suicide constituent, selon nous, le cœur de l'explication du sociologue en ce qu'ils marquent l'ébauche d'une théorie générale des mécanismes sociaux inconscients.

Rappelons d'abord les principaux éléments de cette théorisation qui débute à la page 222 du Suicide. Durkheim vient de conclure sur les trois relations qu'il a mises en évidence (le suicide varie en raison inverse du degré d'intégration de la société religieuse, domestique et politique) et il cherche à présent à expliquer théoriquement ces constats empiriques. Soudainement, il n'y a plus ni classe, ni famille, ni religion, ni âge, ni sexe; seule existe la société. C'est elle qui «souffre», qui «éprouve» des sentiments et qui prend les décisions que les individus ne font qu'appliquer mécaniquement. Ce n'cst pas un hasard si les pages qui suivent déroulent un discours sur l'absence d'épaisseur ontologique de l'individu (à lui seul, [il] n'est pas une fin suffisante pour son activité), et si c'est à ce moment que reparaît la conception durkheimienne de la dualité de l'homme (Mucchielli, 1998a, 167ss.). Par-delà l'homme physique (l'enfant, l'animal), seul compte l'homme social qui est le tout de l'homme civilisé; et c'est lui qui fait le prix de l'exis- 
tence (Durkheim, 1897, 228). Dès lors, toutes les idées morales et religieuses qui se font jour à l'esprit des hommes ne sont que des justifications, des rationalisations d'un sentiment collectif qui a "pénétré » en l'individu. Ces «courants collectifs» ont une autorité qui fait qu'ils s'imposent à l'individu et le poussent avec plus de force encore dans le sens où l'incline déjà l'état de désemparement moral qu'a suscité directement en lui la désagrégation de la société (ibid., 230). Toutes les raisons connues de la conscience individuelle - le divorce, la solitude, la honte, etc. - ne sont que les occasions, les prétextes immédiats, du passage à l'acte. Mais si l'individu cède au moindre choc des circonstances; c'est que l'état où se trouve la société en a fait une proie toute prête pour le suicide (ibid., 230). Certes, chacun aura remarqué que Durkheim consacre plus loin un chapitre aux formes individuelles des différents types de suicides, mais que dit-il en substance? Simplement que chaque suicidé donne à son acte une empreinte personnelle qui exprime son tempérament, les conditions spéciales où il se trouve et qui, par conséquent, ne peuvent être expliquées que par les causes sociales et générales du phénomène (ibid., 312-313). Ainsi, l'individu ne choisit ni sa mort, ni même l'état d'esprit dans lequel il se trouve au moment de mourir. Cet état d'esprit (désespoir, lassitude, irritation, etc.) n'est que le prolongement des causes sociales à l'intérieur de l'individu (ibid., 324). Par exemple, le courant égoïste qui touche un individu le déterminera non seulement à se tuer, mais encore à se sentir mélancolique; de même le courant anomique déterminera chez lui de l'irritation, etc. Et si l'individu présente des sentiments moins nettement définis, associant diverses formes prédéfinies, c'est que les différentes causes sociales du suicide peuvent elles-mêmes agir simultanément sur un même individu et mêler en lui leurs effets. Durkheim retrouve ainsi le postulat exprimé dès les premières pages: les mobiles humains conscients sont trop peu stables et trop peu sûrs pour qu'on puisse fonder sur eux une définition du suicide et une classification de ses différents types.

Quoiqu'il s'en défende dès l'introduction de son livre (ibid., 14, note 1), Durkheim aboutit donc bien à une substantialisation, une hypostase, de la conscience collective (il dit parfois l'ame collective) qui tranche avec les discours des psychiatres. Au terme de son argumentation, il apparaît que l'ultime objet de l'analyse sociologique n'est pas l'explication de la conduite des individus mais celle de l'état de la société, des courants collectifs qui la traversent et agissent automatiquement sur les individus (qui, en pénétrant les individus, les déterminent à se tuer, écrit-il précisément [ibid., 336]). Là se trouve la réalité ultime que le sociologue doit atteindre. C'est ce qu'exprime ce passage connu:

Il y a une humeur collective, comme il y a une humeur individuelle, qui incline les peuples à la tristesse ou à la gaieté, qui leur fait voir les choses sous des couleurs riantes ou sombres. Même, la société est seule en état de porter sur ce que vaut la vie humaine un jugement d'ensemble pour lequel l'individu n'est pas compétent. [...] La société peut, sans sophisme, généraliser le sentiment qu'elle a d'elle-même, de son état de santé et de maladie. Car les individus participent trop étroitement à sa vie pour qu'elle puisse être malade sans qu'ils soient atteints. Sa souffrance devient nécessairement leur souffrance. Parce qu'elle est le tout, le mal se communique aux parties dont elle est faite. [...] Puisque nous sommes son auvre, elle ne peut pas avoir le sentiment de déchéance sans éprouver que, désormais, cette æuuve ne sert plus à rien. Ainsi se forment des courants de dépression et de désenchantement qui n'émanent d'aucun individu en particulier, mais qui expriment l'état de désagrégation où se trouve la société (ibid., 229).

Ce que beaucoup de critiques de l'époque (notamment Simiand, 1898) appelleront à juste titre le réalisme social de Durkheim atteint ici son apogée. Il estime en effet que ces tendances de la société sont des forces aussi réelles que les forces cosmiques, bien qu'elles 
soient d'une autre nature; elles agissent également sur l'individu du dehors, bien que ce soit par d'autres voies (ibid., 349) ${ }^{11}$.

Quel statut doit-on assigner à ces passages de Durkheim? Faut-il pudiquement ignorer leur existence ou bien signaler une réelle faiblesse du sociologue? Ces jugements rétrospectifs sont liées aux enjeux présents de la sociologie. La notion d'erreur est ici, pour l'historien, synonyme d'anachronisme. Nous préférons mettre en avant la rationalité contextuelle de Durkheim en rappelant que sa position relève d'un présupposé cognitif partagé par l'ensemble de la communauté savante de l'époque ${ }^{12}$. On comprendra mieux alors quelle sorte de mécanismes psychologiques il pouvait légitimement imaginer.

\section{Une fascination d'époque pour l'influence des phénomènes inconscients}

Au mois d'aoât 1889 , se déroule à Paris le premier Congrès international de psychologie sous la présidence de Charcot, associant les auteurs français les plus connus (Alfred Espinas, Théodule Ribot, Hippolyte Taine, Charles Richet, Pierre Janet, Valentin Magnan) ainsi que les plus grandes figures internationales des sciences humaines du moment (Wilhelm Wundt, William James, Alexander Bain, Cesare Lombroso, Enrico Morselli, etc.). Au cœur des débats, se situent les discussions sur l'hypnose, les hallucinations, le spiritisme et tous ces «faits extraordinaires» qui fascinent alors les psychologues français les plus positivistes à l'image de leurs collègues anglais pionniers dans ce domaine (Richet, 1890, 33) ${ }^{13}$. Or parmi les auditeurs de ce congrès, mentionnés dans les actes, figurent notamment Tarde, Durkheim et Freud ${ }^{14}$.

Le récit de ce petit événement de la vie intellectuelle parisienne nous permet de mieux faire comprendre la thèse que nous voudrions rapidement développer à présent, à savoir que ce qui s'esquisse en réalité dans ces pages du Suicide, c'est la théorie des représentations collectives de Durkheim, théorie qui est elle-même une reprise, sur un mode original de formalisation, du modèle de causalité psychique dominant dans les sciences humaines à la fin du XIX ${ }^{e}$ siècle: l'inconscient. En effet, les années 1890-1900 constituent en France l'apogée du succès à la fois scientifique et mondain des recherches sur l'hypnose, la suggestion, la double personnalité, tout ce que son ancien camarade normalien Pierre Janet

"Durkheim prolonge du reste la métaphore: de quelque nom qu'on les appelle, ce qui importe, c'est de reconnaître leur réalité et de les concevoir comme un ensemble d'énergies qui nous déterminent à agir du dehors, ainsi que font les énergies physico-chimiques dont nous subissons l'action. Elles sont si bien des forces sui generis, et non des entités verbales, qu'on peut les mesurer, comparer leur grandeur relative, comme on fait pour l'intensité de courants électriques ou de foyers lumineux (ibid., 349). Certes ces réalités ne sont pas visibles, matérialisées, à l'image d'une langue ou d'un code juridique. En réalité, la majeure partie de la conscience sociale resterait diffuse. Elle formerait comme un tourbillon, un magma non encore cristallisé : il y a toute une vie collective qui est en liberté; toutes sortes de courants vont, viennent, circulent dans toutes les directions, se croisent et se mêlent de mille manières différentes et, précisément parce qu'ils sont dans un perpétuel état de mobilité, ils ne parviennent pas à se prendre sous une forme objective (ibid., 355).

12 Sur ces principes méthodologiques, $c f$. aussi notre analyse du cas de l'œuvre de Lombroso (Renneville, 1997b).

13 Pour Richet et Janet, il s'agit explicitement d'imiter la Society for Psychical Research fondée à Londres en 1882 par Henry Sidgwick et qui tentait notamment de recueillir à travers l'Angleterre tous les témoignages ou les auteurs de ces phénomènes psychiques (Parot, 1994).

14 Congrès international de psychologie physiologique, Paris, Bureau des revues, 1890, 5-9. A cette date, Durkheim et Freud ne peuvent naturellement pas se connaître, n'ayant ni l'un ni l'autre publié encore leur premier ouvrage théorique. 
(1889) appelle alors les phénomènes subconscients ${ }^{15}$. Le point commun de cette abondante littérature savante est l'idée que le psychisme est composé de plusieurs dimensions, qu'il existe une dimension cachée, invisible, inconsciente, bien plus déterminante pour l'activité du sujet que la réflexion consciente. Durkheim, qui est issu de la même promotion normalienne que Janet, connaît très bien ces recherches. Si le Suicide ne les mentionne pas explicitement, il y fait allusion à plusieurs reprises. Ainsi:

On s'entend aujourd'hui pour reconnaître que la vie psychique, loin de pouvoir être connue d'une vue immédiate, a, au contraire, des dessous profonds où le sens intime ne pénètre pas et que nous n'atteignons que peu à peu par des procédés détournés et complexes, analogues à ceux qu'emploient les sciences du monde extérieur (Durkheim, 1897, 351).

En réalité, pour comprendre tout le poids de ces conceptions de la vie inconsciente dans la sociologie de Durkheim, il faut se reporter à l'article de 1898 sur les représentations collectives, dont S. Lukes $(1973,6-8)$ - auteur de la biographie de référence sur Durkheim - avait déjà suggéré l'importance. Ce texte capital constitue le véritable prolongement théorique du Suicide (il est du reste écrit dans la foulée). Durkheim se réfère alors avec autorité à Janet et reprend explicitement à son compte la théorie des phénomènes subconscients de ce dernier:

Que des faits bien établis viennent démontrer que la pensée peut se transférer à distance, la difficulté que nous pouvons avoir à nous représenter un phénomène aussi déconcertant ne sera pas une raison suffisante pour qu'on en puisse contester la réalité [...]. Si donc il nous est donné de constater que certains phénomènes peuvent être causés par des représentations, c'est-à-dire s'ils constituent les signes extérieurs de la vie représentative, et si, d'autre part, les représentations qui se révèlent ainsi sont ignorées du sujet en qui elles se produisent, nous dirons qu'il peut y avoir des états psychiques sans conscience, quelque peine que l'imagination puisse avoir à se les figurer. [...] De même, quand on prescrit à un hypnotisé de ne pas voir telle personne ou tel objet qui est sous ses yeux, la défense ne peut agir que si elle est représentée à l'esprit. Cependant la conscience n'en est nullement avertie [...]. Ces expériences ont été faites, il est vrai, sur des états anormaux; mais elles ne font que reproduire sous une forme amplifiée ce qui se passe normalement en nous. Nos jugements sont à chaque instant tronqués, dénaturés par des jugements inconscients; nous ne voyons que ce que nos préjugés nous permettent de voir et nous ignorons nos préjugés (Durkheim, [1898] 1951, 33-35).

Ainsi se trouve enfin explicité, en 1898, ce à quoi Durkheim songe en réalité depuis longtemps. Car comment cette contrainte mentale, cette coercition par laquelle la société imposerait de l'extérieur à l'individu des manières d'agir, de penser et de sentir s'exerce-telle? Comment ces courants suicidogènes agiraient-ils sur les consciences? La réponse était l'inconscient social ${ }^{16}$.

Cet aspect central du livre de Durkheim - le cour de sa conception de la causalité et du rapport individu/société - $\mathbf{a}$ jusqu'à présent été soit jugé anecdotique, soit passé sous silence dans les manuels généraux d'histoire de la sociologie et même dans un manuel consacré exclusivement à la présentation du Suicide (Baudelot, Establet, 1984). Parce qu'ils jugent sans doute cet aspect de la théorie de Durkheim dépassé ou incompréhensible, les sociologues raisonnent comme si l'on pouvait trier le bon grain de l'ivraie, valoriser les raisonnements statis-

15 Sur l'histoire de l'hypnose, et plus largement sur l'omniprésence de ces thèmes dans la psychologie française des années 1880-1900, cf. Barrucand (1967), Carroy (1991) et Ellenberger (1994).

16 La lecture de la théorie durkheimienne des représentations collectives de 1898 à la lumière de la psychologie de l'inconscient a été entrevue pour la première fois à notre connaissance par B. Karsenti (1995). 
tiques de Durkheim et dissimuler l'armature intellectuelle et en particulier le modèle de causalité global au sein desquels ces raisonnements s'exercent. Il nous a semblé préférable d'expliciter la nature de ce cadre global et de le comprendre dans ce que, retournant l'analyse de Durkheim sur lui-même, on peut appeler les représentations collectives de l'époque....

\section{La typologie des suicides: un artifice?}

En conclusion de cette analyse de la conception durkheimienne du rapport individu/société, nous devons revenir sur la typologie de Durkheim pour suggérer que, d'une certaine manière, elle constitue elle aussi une façon de contourner l'approche psychiatrique, de ne pas interroger le rapport pouvant exister entre les facteurs sociaux du suicide et les éventuelles prédispositions individuelles. Sans entrer ici dans le détail de questions qui ont fait l'objet, au moins depuis Parsons, de débats empiriques et théoriques ${ }^{18}$, rappelons les deux types de critiques les plus généralement soulevées.

Tout d'abord, deux des trois (quatre en fait) types de suicide distingués par Durkheim entretiennent des liens bien moins évidents avec les données empiriques, ou du moins avec la signification de ces données, et ne semblent exister que parce qu'ils constituent des référents symétriques ou logiques aux deux autres. Il s'agit des suicides fataliste (opposé à l'anomique) et altruiste (opposé à l'égoïste). On le sait, Durkheim ne consacre qu'une note de bas de page au suicide fataliste qui résulterait de l'excès de réglementation: ce serait le suicide que commettent les sujets dont l'avenir est impitoyablement muré, dont les passions sont violemment comprimées par une discipline oppressive (Durkheim, 1897, 311). Durkheim affirme mais ne démontre pas que ce type n'a qu'un fondement empirique marginal: ce serait le suicide des époux trop jeunes et de la femme mariée sans enfant. Ensuite, il crée un suicide altruiste, auquel il consacre une trentaine de pages, qui correspond d'abord au sacrifice volontaire de soi dans les sociétés primitives ou dans les circonstances de guerre. A nouveau la base empirique est faible et Durkheim ne parviendrait pas à convaincre son lecteur que le sacrifice rituel archaïque ou le sacrifice du soldat au front ont à voir avec le suicide s'il n'assimilait à ces comportements le cas du suicide des militaires ${ }^{19}$. Autrement dit, tout se passe comme si Durkheim avait forgé les suicides altruiste et fataliste dans le seul but de donner une cohérence logique à sa construction d'ensemble.

17 M. Borlandi (1997, 257-258) a récemment rappelé que Pareto partageait le rejet durkheimien des représentations conscientes des acteurs pour fonder un point de vue objectif en sciences sociales. La remarque vaut pour la quasi totalité des auteurs de la fin du XIX' siècle.

18 Sur l'histoire et le point critique de ces débats, $c f$. Ph. Besnard (1987, 65-98). Indiquons simplement le fait que nous rejoignons sur plusieurs points les contributions de A. R. Mawson (1970) et de W. Pope (1976).

19 Durant quinze pages, Durkheim tente en effet d'expliquer que le soldat vivant en caserne se trouve dans une situation de soumission morale au groupe et de dépersonnalisation qui constitue une survivance de la morale primitive. Un courant suicidogène particulier naîtrait dans cette situation et, sous l'influence de cette prédisposition, le soldat se tue pour la moindre contrariété, pour les raisons les plus futiles, pour un refus de permission, pour une réprimande, pour une punition injuste, pour un arrêt dans l'avancement, pour une question de point d'honneur, pour un accès de jalousie passagère ou même, tout simplement, parce que d'autres suicides ont eu lieu sous ses yeux ou à sa connaissance (ibid., 260-261). Ainsi, sans donner le moindre chiffre sur le moindre mobile déclaré, sans s'interroger sur les raisons qui poussent à devenir militaire de carrière, sans même interroger, comme pour les civils, l'état domestique et les convictions religieuses ou politiques des intéressés, Durkheim déduit la signification d'un comportement de la catégorie sociologique globale dans laquelle il range l'individu. Enfin, ainsi que Ph. Besnard (1987, 85) l'a déjà remarqué, Durkheim hésite en réalité sur l'interprétation du suicide des militaires et mêle des éléments relevant dans sa typologie du suicide fataliste (l'excès de régulation); la même hésitation se retrouve dans les quelques lignes consacrées aux suicides des prisonniers (Durkheim, 1897, 394, n. 1). 
Ensuite, il est classique de souligner les problèmes de cohérence que pose la distinction entre les deux grands types de suicide égoïste et anomique. On le sait, ils correspondent pour Durkheim à deux types d'action de la société sur l'individu, l'intégration et la régulation (Besnard, 1987, 93-99) et, derrière cette action, à deux conceptions de l'homme social: l'homme sous influence et l'homme sous contrôle. La difficulté vient du fait que, ainsi que Durkheim l'a lui même reconnu, la distinction est toute relative. Il écrit d'abord qu'il existe entre suicide anomique et suicide égoïste des rapports de parenté du fait que, dans les deux cas, la société n'est pas suffisamment présente aux individus (ibid., 288). Puis il parle d'une affinité spéciale entre les deux types qui ne sont généralement que deux aspects différents d'un même état social (ibid., 325). Enfin et surtout il décrit alors un suicide mixte qui mêle détachement et déréglementation. De fait, les situations concrètes de vie sociale que recouvrent ces deux types ne sont pas très différentes et semblent même parfois compatibles. Si Durkheim ne spéculait pas sur les mécanismes sociaux inconscients, il aurait ainsi pu se demander en quoi le suicide du divorcé ou du veuf diffère radicalement de celui du célibataire? De même, il aurait pu se demander en quoi le renforcement de la tendance globale au suicide en temps de crise ou de croissance économiques subites bouleverse les corrélations établies précédemment entre suicide, religion, famille et politique. Loin de s'exclure, les deux types ne seraient-ils pas au contraire compatibles et cumulables ${ }^{20}$ ?

En fin de compte, n'y aurait-il pas qu'un seul et même suicide, celui du désespoir, simplement influencé de façon plus ou moins forte par tel ou tel facteur social selon le sexe, l'âge, la profession, la période économique, etc.? Du reste, en 1893, dans la Division du travail social, lorsqu'il évoque la question du suicide, Durkheim ne confond nullement suicide et sacrifice. Il ne parle que d'un seul suicide, celui du désespoir qu'il appelle lui-même le vrai suicide:

Le suicide n'apparaît guère qu'avec la civilisation. Du moins, le seul qu'on observe dans les sociétés inférieures à l'état chronique présente des caractères particuliers qui en font un type spécial dont la valeur symptomatique n'est pas la même. C'est un acte non de désespoir, mais d'abnégation [...] le vrai suicide, le suicide triste, est à l'état endémique chez les peuples civilisés (Durkheim, 1902, 226).

La simplicité du constat de 1893 contraste fortement avec les contorsions de 1897. A vrai dire, ces problèmes posés par la démonstration durkheimienne ont commencé par embarrasser ses propres disciples. A tel point que la continuité soulignée par Mauss entre le travail de Durkheim et celui d'Halbwachs peut passer pour un désaveu partiel du premier:

Il serait imprudent, peu scientifique, absurde, quand on se sert du Suicide de Durkheim de ne pas se reporter constamment aux Causes du suicide de M. Halbwachs (Avant-propos, in Halbwachs, 1930, VIII).

\section{L'individuel et le collectif: la solution d'Halbwachs}

Malgré l'avertissement de Mauss, rares sont les sociologues qui considèrent aujourd'hui qu'il est en effet bien peu «scientifique» de lire et de faire lire Durkheim sans lire et faire lire Halbwachs. Du reste, il est significatif que, depuis leur parution en 1930, les

20 Durkheim répond négativement au motif que les deux types de suicide ne concerneraient pas les mêmes milieux sociaux: l'un [égö̈ste] a pour terrain d'élection les carrières intellectuelles, le monde où l'on pense, l'autre [anomique] le monde industriel ou commercial (ibid., 288). Or, à nouveau, cette affirmation n'est pas étayée. Le seul tableau qu'il fournit à l'appui $(X X I V, 286)$ indique des taux de suicide par profession mais ne dit absolument rien sur les motifs ou les facteurs qui toucheraient davantage telle ou telle de ces professions. 
Causes du suicide n'ont jamais été rééditées. Halbwachs posait pourtant, dès l'introduction de son livre, un regard critique sur l'ensemble de la démonstration durkheimienne:

En fermant cet ouvrage [de Durkheim], plus d'un lecteur [...] a sans doute le sentiment que le problème du suicide ne se posait plus, et qu'on en connaissait désormais la solution. Est-ce la dialectique, sont-ce les statistiques qui emportaient la conviction? L'un et l'autre sans qu'on sût bien toujours distinguer ce qui était l'un et ce qui était l'autre; [toujours est-il qu'] on ne s'apercevait pas que l'édifice reposait sur des fondements qui n'étaient point partout aussi solides (Halbwachs, 1930, 3).

L'apport d'Halbwachs se situe sur un triple plan empirique, méthodologique et théorique. Nous ne reviendrons pas ici sur l'ensemble de ces aspects. Nous voulons seulement rappeler d'une part la révision qu'il opère sur la conception de la causalité en sociologie et des rapports entre déterminations individuelles et déterminations sociales, d'autre part le positionnement de la sociologie face à la psychiatrie.

\section{Les motifs et les causes: deux points de vue complémentaires pour analyser un seul et même type de suicide}

Halbwachs conteste à Durkheim la distinction entre les causes véritables du suicide et les prétextes circonstanciels voire illusoires que se donneraient les acteurs pour proposer une autre conception de la causalité. Tout d'abord, Halbwachs ne voit pas en quoi la relative stabilité des motifs de suicide sur le moyen et même sur le long terme serait un indice de leur inexactitude: ce n'est point pourtant par hasard que leur répartition demeure si uniforme. Aime-t-on moins à la ville qu'à la campagne? Souffre-t-on moins de la ruine ou de la honte? Si les motifs sont assez largement les mêmes, n'est-ce pas parce que, au-delà de leurs différences, les genres de vie urbain et rural reposent l'un comme l'autre fondamentalement sur la vie économique et sur la famille? (ibid., 508-509). Ensuite et surtout, Halbwachs pense que l'analyse des motifs n'est nullement un obstacle à l'approche sociologique dès lors que l'on considère le sens social des événements qui provoquent le suicide:

La thèse de Durkheim serait vraisemblable s'il n'existait aucun rapport entre l'action de tels motifs et celle qui résulte de l'ébranlement des sentiments collectifs. Mais il n'en est rien. Lorsqu'on passe en revue les divers motifs particuliers du suicide, on s'aperçoit que, si les hommes se tuent, c'est toujours à la suite d'un événement ou sous l'influence d'un état survenu soit au dehors, soit au dedans (dans leur corps ou dans leur esprit), qui les détache ou les exclut du milieu social, et leur impose le sentiment insupportable de leur solitude. [...] Il n'y a donc pas de différence essentielle entre ce qu'il appelle les motifs et les causes. [...] Ce sont deux états de même nature qui se superposent, ce sont des forces du même genre qui combinent leur action (ibid., 12).

Abandonnant totalement la typologie durkheimienne des suicides ${ }^{21}$, Halbwachs postule donc qu'il n'y a bien partout et quels que soient les motifs immédiats qu'un seul et même sui-

21 Certes, Halbwachs $(1930,451-480)$ reprend à son compte le rapprochement entre suicide et sacrifice, mais dans une perspective toute différente de celle de Durkheim. En réalité il soutient la thèse que, d'un certain point de vue, tout suicide est une forme de sacrifice certes non ritualisé (non reconnu comme tel par les autres) mais vécu comme tout aussi contraignant par l'individu. Dès lors une bonne définition sociologique du suicide doit écarter les formes ritualisées et sacralisées de sacrífice telles qu'on les rencontre dans les sociétés traditionnelles. Halbwachs précise donc la définition de Durkheim: On appelle suicide tout cas de mort qui résulte d'un acte accompli par la victime elle-même, avec l'intention ou en vue de se tuer et qui n'est pas un sacrifice (ibid., 479). 
cide: le suicide du désespoir. Et il ne retient qu'une seule et même théorie de la socialisation: l'intégration sociale. Nulle part, en effet, on ne trouve chez lui les hypothèses de Durkheim sur la nature humaine, ses besoins illimités, sa différence sexuelle naturelle, etc. Ce point est particulièrement intéressant dans l'analyse de l'influence des crises économiques.

En 1897, Durkheim constate, après d'autres, que le suicide s'accroît systématiquement durant les crises économiques. Mais il refuse la «simplicité » de l'explication par la ruine occasionnée pour beaucoup. Son argument est le fait que le suicide croît également en période de prospérité intense, de croissance très rapide. Après des constats statistiques assez sommaires, il met à nouveau en avant une conception de la nature humaine et des besoins illimités et insatiables de l'individu que la société doit régler étroitement, toute perturbation sociale (heureuse ou douloureuse) provoquant inévitablement un dérèglement qui précipite un certain nombre d'individus dans une anomie destructrice (Durkheim, 1897, 272-282). Trente ans plus tard, Halbwachs se montre plus précis dans la définition même d'une crise économique, dans sa mesure et dans celle de ses impacts (Halbwachs, $1930,356 s s$.$) . Le degré d'industrialisation d'un pays lui semble en effet un facteur essentiel$ dans les comparaisons européennes (d'où l'intérêt accordé à l'Allemagne, pays qui s'industrialise fortement à la fin du XIX ${ }^{c}$ siècle). Ensuite, il montre que les accroissements de suicides durant les phases de forte prospérité sont des faits rares et de courte durée, tandis que les phases de dépression ont des effets systématiques et de plus longue durée (ibid., 366ss.). En réalité, la comparaison des rapports des hausses et des baisses des prix sur plus de trente ans montre une opposition parfaite entre le mouvement des prix et le mouvement des taux de suicide, opposition que confirme la comparaison avec le nombre des faillites (ibid., 370-372). Il conclut donc que ce n'est pas la crise comme telle [...], c'est la période de dépression qui suit la crise, qui détermine une augmentation des morts volontaires. Et l'interprétation qu'il propose relève bien de l'intégration, non de la régulation:

Un sentiment obscur d'oppression pèse sur toutes les âmes, parce qu'il y a moins d'activité générale, que les hommes participent moins à une vie économique qui les dépasse, et que leur attention n'étant plus tournée vers le dehors se porte davantage non seulement sur leur détresse ou leur médiocrité matérielle, mais [aussi] sur tous les motifs individuels qu'ils peuvent avoir de désirer la mort (ibid., 374) ${ }^{22}$.

On voit bien ici comment s'articulent chez Halbwachs les causes sociales et les motifs individuels que Durkheim oppose. La même intuition guide du reste Halbwachs dans la critique qu'il reprend et développe à l'encontre des théories psychiatriques déterministes.

\section{D'un déterminisme à l'autre: la causalité psychique simple}

Paradoxalement, si les psychiatres du XIX ${ }^{e}$ siècle étaient dans l'ensemble bien moins réducteurs dans leur explication du suicide que Durkheim ne le disait, le débat causes sociales versus causes bio-psychologiques trouve dans les années trente une actualité certaine. En 1924, dans un livre qui eut un grand succès (L'angoisse humaine), le docteur Maurice de Fleury, membre de l'Académie de médecine, explique en effet que le suicide

22 Il est exact qu'Halbwachs ne souffle mot de l'anomie conjugale (Besnard, 1987, 154). C'est peut-être qu'il préfère passer sous silence les considérations sur l'infériorité biologique des femmes qui sous-tendent les analyses de Durkheim. Par ailleurs, cela ne l'empêche pas de juger capitale la contribution empirique de Durkheim à l'analyse des relations entre suicide et situations familiales (Halbwachs, 1930, 199), ni d'en montrer les limites (ibid., surtout 236-239). 
découle d'une émotion irrésistible, un raptus anxieux, qu'il n'a rien de rationnel et conscient (de Fleury, 1924, 104-105). Reconnaissant que Durkheim a laissé un ouvrage monumental sur la question, il s'empresse de dire que tout a changé depuis un quart de siècle et que c'est à nouveau de la psychopathologie individuelle qu'il faut attendre toutes les lumières (ibid., 110-111). Le paradoxe est que de Fleury utilise ici exactement la même logique argumentative que Durkheim: il estime que les motifs invoqués dans les statistiques ne sont que des circonstances accidentelles formulées dans le langage du sens commun et enregistrées par des fonctionnaires ignorants. Tout comme Durkheim, il entend découvrir le lien caché, la tendance véhémente, commune à tous, cause profonde, véritable, du geste terminal (ibid., 117). Sa solution, c'est un nouveau concept psychiatrique alors en vogue:

Nous savons aujourd'hui - c'est la gloire de Kraepelin, de Kahlbaum, de Deny, de nous l'avoir appris - qu'il s'agit là d'un seul et même déséquilibre mental, constitutionnel, qu'il faut nommer psychose maniaque-dépressive ou cyclothymie (ibid., 123).

A mesure que la vie avance, les sujets atteints de cette maladie connaîtraient des phases dépressives de plus en plus intenses, ce qui expliquerait que le taux de suicide croisse avec l'âge (ibid., 121). Ainsi, seule la psychiatrie apporte une explication satisfaisante de la plupart, sinon de tous les cas de mort voulue (ibid., 131). Le reste n'a aucun intérêt:

Chagrins d'amour ou chagrins de famille, perte d'emploi ou perte de procès, ruine au jeu, souffrances physiques, contrariétés diverses, comme disent nos statistiques, tous ces pauvres petits motifs qui donnent à sourire, n'agissent, ne peuvent déterminer la prodigieuse rupture de l'instinct de conservation, que chez ceux que je viens de dire [sic] (ibid., 124).

En définitive l'épistémologie de Fleury est tout aussi simple et plus péremptoire encore que celle de Durkheim, ses données statistiques étant particulièrement sommaires. Mais la croyance des médecins de l'époque dans l'hérédité des comportements morbides ${ }^{23}$ est si forte, que les plus modérés d'entre eux ne peuvent que reconnaître que les propos de Fleury sont seulement trop exclusifs et que le social et le biologique sont généralement mêlés, le second semblant souvent plus déterminant (Dumas, 1926, 1058).

Ce n'est donc pas sans raison qu'Halbwachs consacre à la discussion des thèses psychiatriques près de soixante-quinze pages de son ouvrage, et que le seul chapitre qu'il en extrait pour le publier séparément est précisément celui-ci (Halbwachs, 1929). Notons pourtant qu'il néglige, comme Durkheim, le fait que la folie est assez souvent, pour les aliénistes, une maladie dont les causes sont à la fois biologiques et sociales. Par ailleurs, dans sa lecture du débat psychiatrique, il ne tient compte ni de la grande diversité des approches médicales du suicide, ni de l'extension contemporaine du champ de la pathologie qui tend alors à dépasser le strict cadre de la clinique aliéniste par le biais de la prophylaxie mentale. Les travaux de S. Serin utilisés par Halbwachs ( $c f$. infra) participent du reste de ce nouveau courant qui relie le suicide à la psychopathologie sans pour autant en faire un acte de folie ${ }^{24}$. Enfin, il est important de noter que, s'il est assez éloigné du champ médical proprement dit, Halbwachs s'adresse par contre directement à des auteurs évo-

23 C'est ce que l'on appelle la doctrine des constitutions développée alors en France par Ernest Dupré (1862-1921), titulaire de la prestigieuse Chaire de clinique des maladies mentales à l'hôpital Saint-Anne, dont de Fleury est un des grands admirateurs.

24 On trouve même dès le tournant du siècle des médecins qui médicalisent le suicide en excluant de leur définition tous les suicides d'aliénés et d'alcooliques. Ils évoquent alors des états de déséquilibre mental causés par l'isolement social ( $c f$. par ex. Hauviller, 1899, 134-136). 
luant dans le même espace social que lui (les facultés de lettres): les psychologues. Halbwachs est même engagé à ce moment, avec Mauss, dans des débats importants avec les psychologues qui portent sur les échanges et les ponts nécessaires entre psychologie et sociologie (Mucchielli, 1994). Charles Blondel et Georges Dumas, tous deux enseignants de psychopathologie, sont ainsi ses principaux interlocuteurs. Et dans ces débats constitués certes d'enrichissements mutuels mais aussi de concurrence et d'enjeux de partages disciplinaires, Halbwachs s'adresse implicitement aux psychologues qui, comme Blondel, durcissent la frontière entre le Normal et le Pathologique pour mieux céder le premier aux sociologues et revendiquer pour eux l'exclusivité du second ( $c f$. Blondel, 1914, 250-251; et par la suite sa réponse à Halbwachs: Blondel, 1933).

Le débat étant appréhendé par Halbwachs dans les limites que nous venons de rappeler, le sociologue partageait le monde psychiatrique entre deux thèses: l'une, modérée, qui associe la dépression et des motifs objectifs issus de la vie sociale et qui reconduit la vieille idée que, pour les suicidés exempts de tares psychopathiques, l'explication sociale garde toute sa valeur (Dumas, 1926, 1058), l'autre, maximaliste (et peut-être dominante dans le monde académique), qui stoppe l'enchaînement des causes à une pathologie spécifique agissant sur l'individu de façon irrépressible.

Face à la première thèse, Halbwachs $(1930,406-407)$ conteste le principe même et la fausse simplicité du débat sur les proportions respectives du biologique et du social dans l'explication du suicide. Pour lui si l'on appelle suicide ces deux catégories d'actes (pathologiques et normaux), c'est bien qu'il s'agit au fond d'une seule et même chose dans notre regard. En réalité, si l'on s'évertue à distinguer sur le papier deux genres de suicide, l'un totalement impulsif, l'autre mûrement réfléchi, l'un bio-psycho-pathologique, l'autre socio-pathologique, c'est que le sens commun aime les distinctions nettes et les types bien tranchés. Or [...] le sens commun a tort de considérer comme typiques ces formes de suicide qui, en réalité, sont rares, sinon tout à fait exceptionnelles. Ce sont des extrêmes, mais la masse des cas se trouve dans l'intervalle, et ne se prête pas à une distinction aussi nette (ibid., 411-412). Il lui semble de meilleure méthode de considérer que le suicidaire est à la fois pleinement pathologique et pleinement normal au plan social: nous admettrions sans peine que chez tout individu qui se tue on trouverait, au moment où il se suicide, et peut-être dans les quelques heures et même les quelques jours qui précèdent, un trouble plus ou moins profond, mais toujours effectif, des fonctions nerveuses et cérébrales, d'où doit résulter un état psychique voisin de ceux que l'on constate dans la névrose d'angoisse, la dépression, etc. En effet, tous les ébranlements soudains dans la vie familiale ou professionnelle, dans la santé, le statut social, etc., toutes ces causes déterminent, à la longue ou brusquement, un état de déséquilibre nerveux. L'opinion commune ne s'y trompe pas: l'on dira de quelqu'un qu'il est égaré par la passion, aveuglé par le chagrin, ivre de fureur, hors de lui, fou de douleur, etc. (ibid., 408) ${ }^{25}$. Que devient la frontière entre les normaux et les malades si la folie est accessible à n'importe quel individu placé dans des conditions de vie insupportables? Il est beaucoup plus sage de considérer que la santé est un équilibre instable, qui comporte bien des oscillations. Ce que l'on observe chez le psychopathe n'est souvent qu'une exagération,

25 Halbwachs peut ici s'appuyer sur Pierre Janet qui qualifiait le suicide de forme morbide de la réaction à l'échec et mettait pareillement en question la frontière entre le normal et le pathologique: On parle trop souvent de la mélancolie comme d'une maladie qui doit se prolonger un certain temps et conserver une certaine unité [...]. Je ne vois pas pourquoi les individus dont parle Durkheim, épuisés par les efforts et les craintes que font naître de mauvaises affaires, par des ruines et des responsabilités terribles, ne tomberaient pas dans des états mélancoliques de courte durée, capables d'amener des suicides considérés à tort comme normaux. Les troubles pathologiques ne doivent pas être séparés des fonctionnements que l'on considère assez arbitrairement comme normaux (Janet, 1928, 369). 
en intensité et en fréquence, de troubles auxquels la plupart des organismes, malgré leur santé apparente sont aussi exposés. Sans doute il y a une différence en ce que le malade est mal adapté aux conditions du milieu normal, qu'il en souffre et que cette souffrance est assez forte pour le pousser dans certains cas jusqu'au suicide. L'homme normal, au contraire est adapté au milieu normal. Mais que le milieu change, pour quelque raison que ce soit, ce sera un milieu anormal, auquel l'homme normal ne sera plus adapté. Il sera dans la situation même où était, et où sans doute est resté le psychopathe (ibid., 410). On arrive ainsi à cette conclusion qui sera saluée par Albert Bayet $(1933,297)$ comme le moyen de sortir du faux débat entre sociologisme et psychologisme qu'aurait instauré Durkheim:

Il ne faut pas croire qu'il y a deux catégories de suicides, dont chacune s'explique par un déterminisme différent, que tantôt, et suivant les individus, c'est le déterminisme organique qui joue, et tantôt le déterminisme social. En réalité le suicide, tout suicide, peut être envisagé de deux points de vue. Suivant que l'on se place à l'un ou à l'autre, on y verra l'effet d'un trouble nerveux, qui relève de causes organiques, ou d'une rupture de l'équilibre collectif, qui résulte de causes sociales (Halbwachs, 1930, 449).

Face à la thèse maximaliste du déterminisme biologique univoque, Halbwachs rappelle d'abord avec ironie que Montesquieu attribuait déjà le suicide des Romains à la coutume et celui des Anglais à un défaut de filtration du suc nerveux. Il constate que les mots ont changé (on dirait désormais un défaut de sécrétion des glandes endoctrines), mais que chez beaucoup de psychiatres l'idée est demeurée aussi simpliste (Halbwachs, 1930, 375) ${ }^{26}$. Or il faut se défier de ces «explications » qui ne reposent en réalité que sur des mots auxquels les psychiatres attribuent une force magique:

Sur quoi se fonde-t-on pour soutenir qu'un malade mental en état de dépression n'est plus sensible à aucune des influences sociales qui s'exercent sur l'homme normal? Suffitil de déclarer qu'il était psychopathe, ou qu'il s'est tué sous le coup de l'ivresse, pour que tout soit dit? (ibid., 435).

Au demeurant, selon leurs propres descriptions, le déprimé ne se tue pas au premier accès de dépression, instantanément, sans avoir eu le temps de réfléchir à sa condition.

L'acte du suicidé ne se produit pas par une sorte de déclenchement automatique. Ce n'est pas un processus purement organique, ni une réaction machinale. Mais il suppose une activité consciente, si confuse soit-elle. Rien ne nous permet d'admettre que le malade ne sait pas qu'il se donne la mort au moment où il se tue (ibid., 436).

Enfin, une fois de plus, tous les mélancoliques ne se tuent pas, et Halbwachs déplore qu'il soit impossible en l'état des statistiques de mesurer l'influence des facteurs familiaux, religieux, sur le suicide de ceux qui sont étiquetés «malades mentaux». Du moins peut-on montrer que la maladie mentale n'est nullement la cause principale des suicides.

\section{Une réfutation empirique de la centralité des cas de psychopathologie}

Tandis que Durkheim et Fleury refusaient toute pertinence à la statistique des motifs, Halbwachs n'a pas besoin de ce déni pour conduire son analyse sociologique. Contre les

26 Il y a là un préjugé populaire qui résiste, au terme duquel le suicide semble un acte si anormal qu'on n'admet pas volontiers qu'il ait pu être accompli par quelqu'un d'autre qu'un malade (ibid., 379). En outre, Halbwachs s'explique cette position par le fait que les psychiatres d'hôpitaux n'observent en effet que les individus gravement malades et qu'ils en tirent des généralisations abusives. 
psychiatres, il accorde à Durkheim le fait que, selon les pays et les moments, le nombre de suicidés déclarés fous varie effectivement beaucoup. Mais il n'en fait pas un argument de principe pour récuser le discours psychiatrique et reconnaît que certains travaux manifestent plus de rigueur méthodologique que d'autres. Ainsi l'enquête menée par le Dr Suzanne Serin en 1925 et 1926 à Paris lui paraît plus sérieuse et plus instructive dans la mesure où les conditions d'enregistrement sont uniformes et où les informations issues d'enquêtes menées systématiquement par le psychiatre auprès des familles des suicidés sont très précises (ibid., 381-383). Serin établit alors le tableau suivant:

Tableau II: Les causes du suicide selon une enquête approfondie de 1926.

\begin{tabular}{|l|c|c|}
\hline Causes & Nombre & $\%$ \\
\hline Chagrin intime & 72 & 17,1 \\
\hline Misère ou revers de fortune & 50 & 11,9 \\
\hline Maladies douloureuses & 44 & 10,5 \\
\hline Total des suicides non psychopathiques & 166 & 39,5 \\
\hline Troubles mentaux bien caractérisés & 78 & 18,6 \\
\hline Déséquilibrés & 72 & 17,1 \\
\hline Suicides de l'ivresse & 130 & 30,1 \\
\hline Total des suicides psychopathiques & 254 & 60,5 \\
\hline Total général & 420 & 100 \\
\hline
\end{tabular}

Source: Serin, 1926, 357-358 (nous calculons les pourcentages) ${ }^{27}$.

Ainsi, quatre suicides sur dix se produisent chez des individus réputés normaux, pour des motifs précis. Inversement seuls $18,6 \%$ des cas relèvent de troubles mentaux bien caractérisés, c'est-à-dire selon le psychiatre, par ordre de fréquence, la dépression mélancolique, les troubles aigus ou subaigus de l'intoxication alcoolique, l'involution sénile, la démence précoce, l'épilepsie, les psychoses traumatiques, l'obsession-suicide, les délires chroniques, les démences organiques, la paralysie générale, la puerpéralité, les délires fébriles, les séquelles d'encéphalite léthargique (Serin, 1926, 359). Si elle n'est que la cause la plus fréquente d'un ensemble qui lui-même n'excède guère $18 \%$ des cas, le premier résultat de cette étude est donc de montrer que la dépression mélancolique n'est pas la cause principale du suicide: elle ne rend compte que d'un suicide sur six (Halbwachs, 1930, 384) ${ }^{28}$.

Ensuite, la catégorie des déséquilibrés correspond selon Serin à des individus qui ne sont ni délirants, ni démentiels, chez qui le suicide a en général des motifs plausibles, mais en disproportion avec cet acte (Serin, 1926, 359). Pour à nouveau un sixième des cas, il est donc clair que l'humeur des suicidés n'est pas seule responsable de leur acte qui a aussi des raisons objectives. De plus, Halbwachs estime qu'il faut ici questionner la distinction entre

27 Note de lecture: Serin a étudié $\mathbf{4 2 0}$ cas mais classé certains suicides d'alcooliques à la fois parmi les déséquilibrés et parmi les suicides de l'ivresse, ce qui explique que le total des suicides psychopathiques soit inférieur à la somme des catégories qui le précèdent.

De même, Serin montrait l'absence (moins de $2 \%$ des cas) de toute hérédité familiale du suicide. 
normal et pathologique. En effet, il est probable que presque tous les suicidés sont plus impressionnables que la moyenne des hommes, puisque, dans les mêmes conditions, un petit nombre d'hommes seulement se tuent, tandis que le plus grand nombre ne se tuent pas. Mais on ne saurait déclarer ces individus fous dans la mesure où on passe en réalité par une série de transitions insensibles des cas anormaux aux cas normaux, de l'impressionnabilité maladive à ce qui n'est qu'une très vive sensibilité. La ligne de séparation qu'on trace entre les uns et les autres est nécessairement arbitraire (Halbwachs, 1930, 386).

Enfin l'alcoolisme est censé expliquer $30 \%$ des suicides décrits comme exécutés sans motifs plausibles, au cours d'accès d'ivresse, en l'absence de tout raptus, de tout épisode onirique, de toute idée délirante, et chez des sujets chez qui ne s'était manifesté aucun signe de déséquilibre (Serin, 1926, 359-360). Or, sur ce point, Halbwachs n'a pas de mal à suggérer que, de ce que le suicidé n'a pas été capable de formuler des motifs précis et ait agi dans un accès d'ivresse, on ne peut en déduire une certitude sur l'absence de motifs (Halbwachs, 1930, 391).

\section{De la statistique aux situations de vie: la sociologie compréhensive d'Halbwachs}

Il faut à notre sens voir dans les Causes du suicide l'esquisse d'une compréhension à la fois sociologique et phénoménologique du suicide:

L'homme est bien obligé de s'adapter au monde, et le paradoxe c'est de vouloir que le monde change sans que lui-même, c'est-à-dire son attitude en face du monde, change aussi (Halbwachs, 1930, 414).

Les hommes dits normaux se suicident toujours après un événement qui a pour effet de les isoler de leur milieu social. Ils peuvent perdre l'affection, l'honneur, la considération, le statut, etc., et ont de ce fait un point commun:

Tous voient s'abaisser leur niveau social. Il sont dans une certaine mesure des déclassés. Or qu'est-ce que se déclasser? C'est passer d'un groupe qu'on connaît, qui vous estime, dans un autre qu'on ignore et à l'appréciation duquel on n'a aucune raison de tenir. On sent alors se creuser autour de soi un vide. Ceux qui vous entouraient autrefois, avec qui vous aviez tant d'idées communes, tant de préjugés en commun, dont tant d'affinités vous rapprochaient parce que vous vous retrouviez en eux comme eux en vous, s'éloignent soudain. Vous disparaissez de leurs préoccupations et de leur mémoire. Ceux au milieu desquels vous vous retrouvez ne comprennent ni votre dépaysement, ni votre nostalgie et vos regrets. Détaché d'un groupe par un ébranlement soudain, vous êtes incapable, ou, du moins, vous vous croyez incapable de retrouver jamais dans un autre quelque appui, ni rien qui remplace ce que vous avez perdu. Mais lorsqu'on meurt ainsi à la société, on perd le plus souvent la principale raison qu'on a de vivre (ibid., 417).

Ainsi, il n'y a bien qu'un seul suicide, celui de l'homme non intégré. Tous ces motifs $s i$ différents qu'ils nous paraissent, ont le même caractère. Ce sont des faits ou des circonstances, des sentiments ou des pensées qui isolent l'homme de la société (ibid., 419). La statistique la plus fine ne fait que vérifier cette évidence que la solitude est la clef de la compréhension aussi bien sociologique que psychologique du suicidé. Et dès lors, pourquoi exclure les individus réputés psychopathes de cette analyse:

N'est-ce pas pour une raison analogue que les délirants et les déprimés se tuent? Certes, à la différence de beaucoup de malades, les psychopathes en période de dépression ne font pas appel à la sympathie compréhensive des autres. Ils cachent plutôt leur maladie et leur souffrance, comme s'ils en éprouvaient quelque honte. Il semble que, comme des 
patients désespérés, ils se sentent d'emblée retranchés du reste du monde. Ce sont bien des isolés. En ce sens [...], ils ne représenteraient qu'un cas particulier d'un phénomène général. Les psychopathes, eux aussi, se tuent parce qu'ils sont en marge de la société et qu'ils ne peuvent plus trouver ailleurs qu'en eux-mêmes un point d'appui (ibid., 421).

En fin de compte, nous jugeons de la raisonnabilité d'un homme du dehors, avec nos normes et notre état de «gens bien portants». Nous qualifions de malades et de délirants des individus qui exagèrent des perceptions, déforment des propos, inventent des agressions ou des hostilités, mais dans leur logique, dans leurs perceptions, ne ressentent-ils pas le même isolement, la même incompréhension, la même solitude:

Ainsi les états psychopathiques produisent le même effet que les autres motifs du suicide. Il n'y a rien qu'une pensée formée par la société soit moins capable de regarder en face que le vide social. C'est cet état d'angoisse et de terreur qui importe seul, et en deçà duquel il n'y a pas lieu de remonter, quand on veut expliquer le suicide. Entre le déprimé psychasthénique, l'homme ruiné, exposé au déshonneur, gravement atteint dans son amour-propre, privé d'un être qu'il aime, il y a sans doute bien des différences, pour qui examine le genre spécial de trouble qui s'est emparé de chacun d'eux. [...] Mais cet état lui-même, c'est-à-dire le sentiment d'une solitude définitive et sans recours, est la cause unique du suicide (ibid., 425).

Et ce sentiment est bien encore et toujours proprement social puisque c'est devant le miroir de notre vie-avec-les-autres qu'il se détermine négativement:

Le désespéré réfléchit, il interroge silencieusement les êtres et les choses qui l'entourent, il reçoit des réponses négatives et décourageantes qui ne sont que l'écho de sa tristesse, et il les interprète comme un encouragement à quitter la vie (ibid., 469).

***

Le Suicide est rédigé et publié à un moment difficile de la carrière de Durkheim. Sa volonté d'établir la sociologie comme domaine de recherche autonome et indépendant par une méthodologie explicite s'est soldée jusqu'ici par un échec, la réception des Règles ayant été particulièrement mauvaise (Paoletti, 1995). Cette étude est une manière d'imposer autrement - sur un cas concret et parfaitement choisi - cette césure fondatrice. C'est sans doute pourquoi Durkheim n'a pas adopté une attitude conciliatrice et qu'il a cherché au contraire à critiquer systématiquement les sciences déjà établies qui traitaient d'objets que le sociologue estimait être de la seule compétence de sa méthode. Nous avons tenté de montrer que c'est cette stratégie générale qui introduit chez Durkheim un biais cognitif dans la lecture des psychiatres de son temps. Dans son raisonnement, Durkheim était pourtant beaucoup plus proche d'eux qu'il ne le concédait. Ils partageaient notamment l'idée que le taux de suicide était, dans la société française de l'époque - et donc pour des raisons sociales -, pathologique. Les médecins ne s'étant par ailleurs jamais interdit de faire à leur manière de la «sociologie», les deux domaines étaient ainsi en concurrence directe. Le projet de Durkheim ne résidait pas dans l'alliance des compétences mais dans l'érection de frontières strictes: aux médecins les pathologies individuelles, aux sociologues les pathologies sociales. Le dessein était ambitieux, sa réalisation délicate. Le sociologue devait proposer en effet une conception alternative des relations individu-société, qui ne passât plus par la métaphore organiciste et des analogies causales entre les pathologies individuelles et collectives. Parvint-il toujours à se défaire des représentations médicales? On peut en douter, lorsque l'on considère ses allusions récurrentes et non critiques au concept de neurasthénie. 
Quoi qu'il en soit, l'innovation introduite par Durkheim en 1897 dans l'histoire des analyses du suicide n'est pas empirique, ni même méthodologique (les variations concomitantes), mais bien théorique. Elle tient tout particulièrement à son refus de s'interroger sur les motifs individuels du suicide. En effet, s'ils s'en tenaient, faute de sources, à un niveau de corrélations très général, les premiers statisticiens regrettaient, à l'instar de Quetelet $(1835,417)$, de manquer d'informations sur les motifs qui déterminent l'homme au suicide, informations qu'ils auraient souhaité relier aux observations chiffrées. C'est cette lacune que les psychiatres tentèrent ainsi de combler dans les décennies suivantes en analysant les procès-verbaux des suicidés pour dresser la liste des causes possibles, incluant des «facteurs sociaux». En 1897, Durkheim disposait par contre de séries statistiques longues et de plusieurs milliers d'études de cas dont il aurait pu faire usage. Il ne l'a pourtant pas fait pour au moins deux raisons:

- premièrement parce que son travail visait d'une part, dans l'ensemble du champ intellectuel à auto-instituer la pertinence de la rupture épistémologique fondatrice qu'il prétendait réaliser par la sociologie, d'autre part, face surtout à Tarde, à imposer la supériorité de sa conception de la sociologie dans un contexte de concurrence;

- deuxièmement parce que ses croyances et ses préjugés (quant aux phénomènes inconscients, quant aux besoins de régulation du psychisme humain, quant à la vertu de la pauvreté, etc.) ont souvent guidé son analyse. De ce point de vue, Durkheim apparaît moins comme le premier des sociologues que comme le dernier des philosophes. La preuve en est qu'Halbwachs et toute la sociologie du suicide après Durkheim a abandonné non pas ses principes méthodologiques mais bien sa construction théorique (la typologie des suicides et les préjugés en question).

Loin de déconsidérer l'œuvre, ces limites, replacées dans leur contexte historique, lui donnent en un sens un nouvel intérêt pédagogique. Plutôt que de sacraliser la parole du «fondateur de la sociologie française», on pourrait alors expliquer aux étudiants pourquoi et comment Durkheim a pu transgresser les principes qu'il avait lui-même énoncés deux ans plus tôt dans les Règles de la méthode sociologique.

Laurent Mucchielli CNRS, Centre de Recherches Sociologiques sur le Droit et les Institutions Pénales

Immeuble Edison 43, bd Vauban, F-78280 Guyancourt.

Marc Renneville Institut d'Histoire de la Médecine et de la Pharmacie Université Paris V 24, rue du Faubourg Saint-Jacques, F-75014 Paris.

\section{BIBLIOGRAPHIE}


BAECHLER J., Les suicides, Paris, Calmann-Lévy, 1975.

BARRUCAND D., Histoire de l'hypnose en France, Paris, PUF, 1967.

BAUDELOT C., ESTABLET C., Durkheim et le suicide, Paris, PUF, 1984.

BAYET A., C.-r. de HALBWACHS (1930) et de BLONDEL (1933), Revue Philosophique, 1933, 2, 295-298.

BEARD G. M., Neurastenia, or Nervous Exhaustion. Its Symptoms, Nature, Consequences, Treatment, New York, W. Wood \& Company, 1869.

BERRIOS G. E., MOHANNA M., Durkheim and French Psychiatric Views on Suicide during the 19th Century. A Conceptual History, British Journal of Psychiatry, 1990, 156, 1-9.

BERTHELOT J.-M., 1895, Durkheim. L'avènement de la sociologie scientifique, Toulouse, Presses universitaires du Mirail, 1995.

BERTILLON J., De l'influence du mariage sur la tendance au suicide, Bulletins de la Société d'Anthropologie de Paris, 1880, 3, 277-295.

BERTILLON J., Etude démographique du divorce et de la séparation de corps dans les différents pays d'Europe, Annales de Démographie Comparée, 1882, 257-451.

BESNARD P., Durkheim et les femmes ou Le suicide inachevé, Revue Française de Sociologie, 1973, 14, 1 , 27-61.

BESNARD P., Anti- ou anté-durkheimisme? Contribution au débat sur les statistiques officielles du suicide, Revue Française de Sociologie, 1976, 17, 2, 313-341.

BESNARD P., L'anomie. Ses usages et ses fonctions dans la discipline sociologique depuis Durkheim, Paris, PUF, 1987.

BLONDEL C., La conscience morbide. Essai de psychopathologie générale, Paris, Alcan, 1914.

BLONDEL C., Le suicide, Strasbourg, Librairie Universitaire d'Alsace, 1933.

BORLANDI M., L'individuel et le social, in CUIN C.-H., Ed., Durkheim d'un siècle à l'autre. Lectures actuelles des «règles de la méthode sociologique», Paris, PUF, 1997, 249-264.

BORLANDI M., MUCCHIELLI L., Eds, La sociologie et sa méthode. Les règles de Durkheim un siècle apres, Paris, L'Harmattan, 1995.

BOURDIN C. E., Du suicide considéré comme maladie, Paris, De Hunneyer et Turpin, 1845.

BOURDIN C. E., Le suicide est-il toujours le résultat ou, si l'on veut, le symptôme d'un trouble de l'esprit? En d'autres termes, le suicide est-il toujours une maladie?, Annales Médico-psychologiques, 1846a, 8, 41-55.

BOURDIN C. E., Le suicide est-il une maladie? Quelques mots sur cette question à MM. Les rédacteurs des Annales d'hygiène, Annales d'Hygiène Publique et de Médecine Légale, 1846b, 36, 465-469.

BOUVERET L., La neurasthénie. Epuisement nerveux, Paris, J.-B. Baillière, 1891.

BRIERRE DE BOISMONT A., C.-r. D'ETOC-DEMAZY (1844), Annales Médico-psychologiques, 1844, 3, 495-501.

BRIERRE DE BOISMONT A., Quelques remarques sur le suicide à l'occasion de la brochure de M. Bourdin, Annales d'Hygiène Publique et de Médecine Légale, 1846, 35, 423-441.

BRIERRE DE BOISMONT A., Du suicide et de la folie suicide considérés dans leurs rapports avec la statistique, la médecine et la philosophie, Paris, Baillière, 1865 (1856).

CARROY J., Hypnose, suggestion et psychologie. L'invention de sujets, Paris, PUF, 1991.

CHARCOT J. M., Leçons du mardi à la Salpêtrière - Policlinique 1887-88, 1888-89, Paris, Bureaux du Progrès médical, 2 vol., 1890-1892.

CHESNAIS J.-C., Les morts violentes en France depuis 1826, Cahiers de l'INED, n75, Paris, PUF, 1976.

CHESNAIS J.-C., Histoire de la violence en Occident de 1800 à nos jours, Paris, Robert Laffont, 1981.

CHEVREY J., Essai médical sur le suicide, considéré, dans tous les cas, comme le résultat d'une aliénation mentale, thèse de médecine, Paris, $n^{\circ} 128,1816$. 
DELASIAUVE L., Lettre à $M$. le rédacteur du journal L'Observation en réponse au dernier article de M. Bourdin sur le suicide, Paris, 1850.

DESROSIERES A., La politique des grands nombres. Histoire de la raison statistique, Paris, La Découverte, 1993.

DOUGLAS J., The Social Meanings of Suicide, Princeton, Princeton University Press, 1967.

DUMAS G., C.-r. de DE FLEURY (1924), Journal de Psychologie, 1926, décembre, 1053-1060.

DURKHEIM E., Suicide et natalité. Etude de statistique morale, Revue Philosophique, 1888, 26, 446-463.

DURKHEIM E., Le suicide. Etude de sociologie, Paris, Alcan, 1897.

DURKHEIM E., Représentations individuelles et représentations collectives, Revue de Métaphysique et de Morale, 1898, 6, 273-302. Repr. in Sociologie et Philosophie, Paris, P.U.F., 1951, 13-50.

DURKHEIM E., De la division du travail social, Paris, Alcan, 1902 (1893).

DURKHEIM E., Sociologie religieuse et théorie de la connaissance, Revue de Métaphysique et de Morale, 1909, 17, 733-758.

DURKHEIM E., C.-r. de A. KROSE (Die ursachen der selbstmordhäufigkeit, Feiburg, Herder, 1906), Année Sociologique, 1910, 11, 511-515.

DURKHEIM E., L'éducation morale, Paris, Alcan (cours de 1899), 1925.

DURKHEIM E., Les règles de la méthode sociologique, Paris, PUF, 1947 (1895).

DURKHEIM E., Leçons de sociologie. Physique des maeurs et du droit, Paris, PUF (cours de 1890-1900), 1950.

EBRARD N., Du suicide, considéré aux points de vue médical, philosophique, religieux et social, Avignon, Séguin, 1870.

ELLENBERGER H., Histoire de la découverte de l'inconscient, Paris, Fayard, 1994 (1974).

ETOC-DEMAZY G.-F., Recherches statistiques sur le suicide, appliquées à l'hygiène publique et à la médecine légale, Paris, Germer Baillière, 1844.

ETOC-DEMAZY G.-F., Sur la folie dans la production du suicide, Annales Médico-psychologiques, 1846, 7, 338-362.

de FLEURY M., L'angoisse humaine, Paris, Les Editions de France, 1924.

HALBWACHS M., Le suicide et les maladies mentales, Revue Philosophique, 1929, 108, 321-360.

HALBWACHS M., Les causes du suicide, Paris, Alcan, 1930.

HAUVILLER P., Du suicide. Unité de son étiologie. Son traitement, Thèse de médecine ( $\left.{ }^{\circ} 169\right)$, Paris, Jouve et Boyer, 1899.

JANET P., L'automatisme psychologique. Essai de psychologie expérimentale sur les formes inférieures de l'activité humaine, Paris, Alcan, 1889.

JANET P., De l'angoisse à l'extase, vol. 2 (Les sentiments fondamentaux), Paris, Alcan, 1928.

KARSENTI B., La spécificité psychologique de la sociologie. De Durkheim à Mauss, in BORLANDI M., MUCCHIELLI L., Eds, La sociologie et sa méthode. Les règles de Durkheim un siècle après, Paris, L'Harmattan, 1995, 297-320.

LECUYER B.-P., Médecins et observateurs sociaux: les Annales d'Hygiène Publique et de Médecine Légale (1820-1850), in Pour une histoire de la statistique, Paris, Economica-INSEE, 1977, vol. 1, 445-476.

LEGOYT A., Le suicide ancien et moderne. Etude philosophique, morale et statistique, Paris, A. Drouen, 1881.

LE MEE R., La statistique démographique officielle de 1815 à 1870 en France, Annales de Démographie Historique, 1979, 251-278.

LE ROY E., Etude sur le suicide et les maladies mentales dans le département de Seine-et-Marne avec points de comparaison pris en France et à l'étranger, Paris, Masson et fils, 1870.

LISLE E. P., Du suicide. Statistique, médecine, histoire et législation, Paris, Baillière, 1856. 
LUKES S., Emile Durkheim. His Life and Work. A Historical and Critical Study, London, Allen Pane, 1973.

MAWSON A. R., Durkheim and Contemporary Social Pathology, British Journal of Sociology, 1970, 21, 3 , 298-311.

MOLEON J.-G.-V., Rapports généraux sur la salubrité publique, Paris, Bureau du recueil industriel, 1829.

MORSELLI E., Il suicidio. Saggio di statistica morale comparativa, Milano, Fratelli Dumolard, 1879.

MUCCHIELLI L., Psychologie et sociologie en France, l'appel à un territoire commun: vers la psychologie collective (1890-1940), Revue de Synthèse, 1994, 3-4, 445-483.

MUCCHIELLI L., La découverte du social. Naissance de la sociologie en France (1870-1914), Paris, La Découverte, 1998a.

MUCCHIELLI L., De l'inscription biographique des découvertes savantes à la notion de Névrose créatrice. La "révélation» d'Emile Durkheim, in CARROY J., RICHARD N., Eds., Les récits de découverte en sciences humaines, Paris, L'Harmattan, 1998b (sous presse).

NYE R., Heredity, Pathology and Psychoneurosis in Durkheim's Early Work, Knowledge and Society. Studies in the Sociology of Culture, 1982, 4, 103-142.

PAOLETTI G., La réception des Règles du vivant de Durkheim (1894-1917), in BORLANDI M., MUCCHIELLI L., Eds, La sociologie et sa méthode. Les règles de Durkheim un siècle après, Paris, L'Harmattan, 1995, 247-284.

PAROT F., Le bannissement des esprits. Naissance d'une frontière institutionnelle entre spiritisme et psychologie, Revue de Synthèse, 1994, 3-4, 417-443.

PETIT J.-B., Recherches statistiques sur l'étiologie du suicide, thèse de médecine, Paris, $n^{\circ} 115,1850$.

PEUCHET J., (1836), Du suicide et de ses causes, repr. in MARX K., PEUCHET J., A propos du suicide, Paris, Climats, 1992, 33-98.

POPE W., Durkheim's «Suicide». A Classic Analysed, Chicago, University of Chicago Press, 1976.

QUETELET A., Sur l'homme et le développement de ses facultés, ou essai de physique sociale, Paris, Bachelier, 1835.

RENNEVILLE M., La médecine du crime. Essai sur l'émergence d'un regard médical sur la criminalité en France, 1785-1885, Lille, ANRT, Presses Universitaircs du Septentrion, 1997a.

RENNEVILLE M., Rationalité contextuelle et présupposé cognitif. Réflexion épistémologique sur le cas Lombroso, Revue de Synthèse, 1997b, 4, 495-529.

RICHET C., Les travaux du Congrès de psychologie physiologique, in Congrès international de psychologie physiologique, Paris, Bureau des revues, 1890.

SERIN S., Une enquête médico-sociale sur le suicide à Paris, Annales Médico-psychologiques, 1926, 2, 356363.

SIMIAND F., L’année sociologique 1897, Revue de Métaphysique et de Morale, 1898, 6, 608-653.

TIBERI A., Le suicide dans l'hérédité morbide, thèse de médecine, Lyon, ${ }^{\circ} 1124,1895$.

\section{Summary}

The article questions the link between individuals and society and the ideas of psychological causality set out by Durkheim in Suicide, and puts them into historical perspective, considering previous readings and the critical revisionist perspective by Halbwachs in 1930. First, we consider the ways in which Durkheim interpreted psychiatric theories which explained social facts through individual pathology. We then show that this Durkheimian construction involves the rejection of individual motive and replaces it by a different psychological interpretation which is based on socially determined unconscious mechanisms. We underline the fact that this theoretical construction had several difficulties even in relation to the empirical findings of the period and that it leads us to question the Durkheimian typology of suicide. Finally, we consider the way in which, 
some 30 years later, Halbwachs was able largely to resolve these difficulties by abandoning the theoretical axioms which Durkheim had placed at the heart of sociological analysis.

KEY-WORDS: SUICIDE - SOCIOLOGICAL THEORY - HISTORY OF PSYCHIATRY - HISTORY OF SOCIOLOGY - EPISTEMOLOGY

\section{Zusammenfassung}

Dieser Artikel behandelt in historischer Perspektive die Beziehung zwischen Individuum und Gesellschaft sowie das Modell psychischer Ursachen, wie es von Durkheim in seiner Selbstmordstudie entwickelt wurde. Dazu wird dieses Modell einerseits in Beziehung gesetzt zum Stand der Interpretationen, die ihm vorausgingen und andererseits zu einer kritischen Lesart, wie sie von Halbwachs 1930 entwickelt worden ist. Zunächst gehen wir im Detail auf die Analyse von Durkheim ein, in der er psychiatrische Thesen behandelt, die für ihn eine soziale Tatsache über individuelle Pathologien erklären. Wir zeigen dann, da $\beta$ die durkheimsche Konstruktion eine Zurückweisung individueller Motive impliziert, welche durch eine andere psychologische Interpretation ersetzt werden, die sich auf sozial determinierte unbewußte Mechanismen gründet. Dabei unterstreichen wir, daß diese theoretische Konstruktion auf einige Schwierigkeiten bei der Konfrontation mit den empirischen Daten der damaligen Zeit stößt und den Wert der durkheimschen Selbstmordtypologie grundsätzlich in Frage stellt. Schließlich untersuchen wir die Art, in der es Halbwachs dreißig Jahre später gelang, diese Probleme eher zu lösen, indem er auf die theoretischen Postulate, die Durkheim ins Zentrum der soziologischen Analyse stellte, verzichtete.

\section{Samenvatting}

De auteurs van dit artikel bestuderen de verhouding individu / maatschappij alsook het psychische causaliteitsmodel zoals bedacht door Durkheim in zijn werk «Le Suicide». Ze plaatsen dit werk in een historisch perspectief ten aanzien van enerzijds de interpretaties (gedachtegoed) die het werk vooraf gingen en anderzijds vertrekkende van de kritische herlezing verricht door Halbwachs in 1930. In eerste instantie wordt door de auteurs in detail de manier besproken waarop Durkheim de psychiatrische stellingen afweert. Stellingen die volgens hem een sociaal feit verklaren door individuele pathologie (ziektenkunde). Vervolgens wordt aangetoond dat de opstelling van Durkheim de verwerping van de analyse van individuele motieven impliceert en deze theoretische constructie met een aantal tegenkantingen te maken krijgt als ze wordt geconfronteerd met empirische gegevens uit de tijd. Uiteindelijk brengt dit met zich mee dat men zich vragen gaat stellen inzake de gegrondheid van Durkheim's zelfmoordtypologie. Tenslotte wordt in het artikel de manier bestudeerd waarop meer dan 30 jaar later Halbwachs erin slaagt deze moeilijkheden op te lossen door afstand te nemen van de theoretische axioma's die Durkheim had geplaatst in het kader van de sociologische analyse. 